\title{
ON GALOIS CONDITIONS AND GALOIS GROUPS OF SIMPLE RINGS $\left({ }^{1}\right)$
}

\author{
BY \\ TAKASI NAGAHARA
}

Throughout the present paper, $R$ will be a simple ring, where we shall understand by a simple ring a total matrix ring over a division rings. If $S^{\prime}$ is any subring containing the identity element 1 of $R$, we denote by $V_{R}\left(S^{\prime}\right)$ the centralizer of $S^{\prime}$ in $R, V_{R}^{2}\left(S^{\prime}\right)=V_{R}\left(V_{R}\left(S^{\prime}\right)\right)$ and by $(S)\left(S^{\prime}, R\right)$ we denote the group of all automorphisms of $R$ which are the identity on $S^{\prime}$. We shall be concerned with a fixed $S^{\prime}$ which is denoted by $S$ and shall consider primarily subrings $S^{\prime}$ of $R$ which contain $S$. We abbreviate $V_{R}(S)=V, V_{R}^{2}(S)=H, \&(S, R)=\mathbb{B}$. A subring $S^{\prime}$ of $R$ is said to be regular when $S^{\prime}$ and $V_{R}\left(S^{\prime}\right)$ are both simple. Further, if $S^{\prime}$ is regular and coincides with fixed ring $J\left(S_{(S)}\left(S^{\prime}, R\right), R\right)$ of $\Theta^{\prime}\left(S^{\prime}, R\right)$ in $R$, then we say that $R$ is Galois over $S^{\prime}$. In particular, if $R$ is Galois over $S$ and $V$ coincides with the center of $R$ then we say that $R$ is outer Galois over $S$. If, for each finite subset $F$ of $R$, the ring $S[F]$ generated by $S$ and $F$ is a finitely generated left $S$-module, then we say that $R$ is left locally finite over $S$. If $S^{*}$ is a simple subring containing the identity element of $R$ then, as is well known, any subring $T$ of $R$ containing $S^{*}$ contains a linearly independent left (right) basis over $S^{*}$. By $\left[T: S^{*}\right]_{l}\left(\left[T: S^{*}\right]_{r}\right)$ denote the left (right) dimension. In case $\left[T: S^{*}\right]_{l}=\left[T: S^{*}\right]_{r}$, then they are denoted by $\left[T: S^{*}\right]$. If $M$ is any subset of $R$, we denote by $M_{l}\left(M_{r}\right)$ the set of left (right) multiplications determined by elements of $M$. For any regular element $a$ of $R$, we shall denote by $\langle a\rangle$ an inner automorphism $a_{l} a_{r}^{-1}$ of $R$ and by $\langle M\rangle$ denote the set of inner automorphisms determined by regular elements of $M$. In our papers cited in the references, $\langle M\rangle$ has been denoted as $\widetilde{M}$. We shall understand by a $\operatorname{Hom}(R, R)$-module $M$ a right $\operatorname{Hom}(R, R)$ module $M$. For any subset $\mathfrak{F}$ of $\operatorname{Hom}(R, R)$, and for any subset $M$ of $R$, we denote by $\mathfrak{F} \mid M$ the restriction of $\mathfrak{F}$ to $M$ and by \# $(\mathfrak{F} \mid M)$ denote the cardinal number of $\mathfrak{F} \mid M$. We shall consider the following conditions:

$\left(\mathrm{A}_{l}\right)$ : (i) $S$ is regular and $\Theta R_{r}$ is dense in $\operatorname{Hom}_{S_{l}}(R, R)$ in the finite topology, and

(ii) $R$ is left locally finite over $S$.

(B) : (i) $R$ is Galois over $S$ and $R$ is $S_{l} V_{l}-R_{r}$-irreducible, and

Received by the editors December 1, 1963.

( ${ }^{1}$ ) This work was carried out partly under the support of NSF grant GP 1649. 
(ii) $R$ is left locally finite over $S$.

$\left(\mathrm{C}_{l}\right):$ (i) $R$ is Galois over $S$ and $R$ is $S_{r} V_{r}-R_{l}$-irreducible, and

(ii) $R$ is left locally finite over $S$.

$\left(\mathrm{D}_{l}\right):$ (i) $R$ is Galois over $S, H$ is simple, and $\left[V_{R}^{2}\left(S^{\prime}\right): H\right]_{l}=\left[V: V_{R}\left(S^{\prime}\right)\right]_{r}$ for every regular subring $S^{\prime}$ with $\left[S^{\prime}: S\right]_{l}<\infty$, and

(ii) $R$ is left locally finite over $S$.

$\left(\mathrm{E}_{l}\right)$ : (i) $S$ is regular and $R$ is Galois over every regular subring $S^{\prime}$ with $\left[S^{\prime}: S\right]_{l}<\infty$, and

(ii) $H$ is simple and $\left[R^{\prime}: H\right]_{l}=\left[V: V_{R}\left(R^{\prime}\right)\right]_{r}$ for every regular subring $R^{\prime}$ containing $H$ with $\left[R^{\prime}: H\right]_{l}<\infty$, and

(iii) $R$ is left locally finite over $S$.

$\left(\mathrm{E}_{l}^{*}\right):\left(\mathrm{E}_{l}\right)$ plus the conditions that $V_{R}^{2}\left(S^{\prime}\right)$ is simple and $V_{R}^{2}\left(R^{\prime}\right)=R^{\prime}$.

We shall also consider conditions $\left(\mathrm{A}_{r}\right)-\left(\mathrm{E}_{r}^{*}\right)$ obtained by interchanging $l$ and $r$, and left and right in $\left(\mathrm{A}_{l}\right)-\left(\mathrm{E}_{l}^{*}\right)$.

Recently in [4], the present author and $H$. Tominaga succeeded in constructing a Galois theory of simple rings under the condition $\left(\mathrm{E}_{l}^{*}\right)$ which we called "hereditarily Galois (abbreviated, h-Galois) and left locally finite." That theory contained the theory of Walter [10] since Galois extensions of division rings satisfy $\left(E_{l}^{*}\right)$. In [5], we also presented some useful results under the condition $\left(A_{l}\right)$. One of our purposes is to prove that the conditions $\left(A_{l}\right)-\left(E_{r}^{*}\right)$ are all equivalent. Another one is to characterize regular subgroups of $(B)$, which will be defined later, and we shall present a more desirable Galois correspondence than that of [4] under the conditions $\left(\mathrm{A}_{l}\right)-\left(\mathrm{E}_{r}^{*}\right)$.

The next propositions $(\alpha, \beta$, and $\gamma)$ will play important roles. We also require the fundamental theorem of simple rings and the fundamental theorem of [4] for our last theorem. Otherwise, this paper is selfcontained.

Proposition $\alpha$. Let $R$ satisfy $\left(\mathrm{A}_{l}\right)$ over $S$. Then, we have:

(i) If $S^{\prime}$ is a simple subring of $R$ with $\left[S^{\prime}: S\right]_{l}<\infty$, then $R$ is completely $S_{l}^{\prime}-R_{r}$-reducible. If, in addition, $V_{R}\left(S^{\prime}\right)$ is a division ring, then $R$ is $S_{l}^{\prime}-R_{r^{-}}$ irreducible [5, Lemma 2].

(ii) If $T$ is a subring of $R$ left finite over $S$ such that $R$ is $T_{l}$ - $R_{r}$-irreducible, then $J(S)(T, R), R)=T[5$, Lemma 1$]$.

(iii) If $S^{\prime}$ is a regular subring of $R$ with $\left[S^{\prime}: S\right]_{l}<\infty$, then $R$ is Galois over $S^{\prime}$ [5, Theorem 2].

Proposition $\beta$. If $(5)$ is a finite group of outer automorphisms of $R$, then $J\left(S^{*}, R\right)$ is simple and $R$ is finite and outer Galois over $J\left(\xi^{*}, R\right)$ (and $\left(S^{\prime}\left(J\left(\xi^{*}, R\right), R\right)-\left(\xi^{*}\right)[1]\right.$. In general, if $R$ is outer Galois and left locally finite (or right locally finite) over $S$, then every subring $S^{\prime}$ containing $S$ is simple, and $R$ is outer Galois and two-sided locally finite over $S^{\prime}$. If $S^{*}$ is a subgroup of $\mathscr{H}^{S}$ such that $J\left(\mathcal{S}^{*}, R\right)=S$, and if $\left[S^{\prime}: S\right]_{l}<\infty$, then $\left.J(\circlearrowleft)\left(S^{\prime}, R\right) \cap()^{*}, R\right)=S^{\prime},\left[S^{\prime}: S\right]_{l}=\left[S^{\prime}: S\right] r=\#\left(\left(^{*} \mid S^{\prime}\right)\right.$, and $S^{\prime}=S[a]$ 
for some $a \in S^{\prime}[2]$, [3, Theorem 1.1 and Corollary 1.4], [4, Lemma 1.8], and $[9]$.

REmark. If $R$ is Galois over $S$, then $H \sigma=H$ for any $\sigma \in \mathbb{( S )}$, and $J(\$ \mid H, H)=S$. In addition, if $H$ is simple and $H$ is left locally finite (or right locally finite) over $S$, then $H$ is outer Galois over $S$.

Proposition $\gamma$. Let $R$ be Galois over $S, H$ simple, and $H$ left locally finite (or right locally finite) over $S$. Let $T$ be a subring of $R$ left finite over $S$ such that $R$ is $T_{l}$ - $R_{r}$-irreducible. Then we have:

(i) $H \cap J(S(T, R), R)$ is finite over $S[5$, Lemma 5].

(ii) If $[T: H \cap T]_{l}=\left[V: V_{R}(T)\right]_{r}$, then

$$
\left.\left.\operatorname{Hom}_{S_{l}}(T, R)=(\$) T\right) R_{r}=(\$) \mid T\right) R_{r} \quad \text { and } \quad(S \mid T=(S * \mid T
$$

for any subgroup $\left(S *\right.$ of $(S)$ such that $\left(S * \supseteq\langle V\rangle\right.$ and $J\left(S^{*}, R\right)=S$. In particular, if $H \cap T=S$ and $[T: S]_{l}=\left[V: V_{R}(T)\right]_{r}$, then $\operatorname{Hom}_{S_{l}}(T, R)=(\langle V\rangle \mid T) R_{r}$ and $\Theta|T=\langle V\rangle| T$ (its proof is similar to that of $[4$, Lemma 3.1]).

Throughout this paper, we shall use the following conventions: $R$ $=\sum D e_{i j}$, where $e_{i j}$ 's are matrix units and $D=V_{R}\left(\left\{e_{i j}\right\}\right)$ is a division ring. In case $V\left(=V_{R}(S)\right)$ is a simple ring, we set $V=\sum U g_{p q}$, where the $\boldsymbol{g}_{p q}$ 's are matrix units and $U=V_{V}\left(\left\{g_{p q}\right\}\right)$ is a division ring.

In case $H\left(=V_{R}^{2}(S)\right)$ is a simple ring, we set $H=\sum K d_{h k}$, where the $d_{h k}$ 's are matrix units and $K=V_{H}\left(\left\{d_{h k}\right\}\right)$ is a division ring.

$C$ will be the center of $R$, and $C_{0}$ the center of $V$, which coincides with the center of $H$.

1. On Galois conditions. Now, we shall begin our study with the following lemma.

Lemma 1. Let $P$ and $Q$ be subrings of $R$ containing 1 such that $x y=y x$ for every $x$ in $P$ and every $y$ in $Q$. If $R$ is $P_{l} Q_{l}-R_{r}$-irreducible, then we have:

(i) $R$ is a completely reducible homogeneous $P_{l}-R_{r}$-module.

(ii) $V_{R}(P)$ is a simple ring; if $V_{R}(P)$ is a division ring then $R$ is $P_{l}-R_{r^{-}}$ irreducible.

(iii) $\left[V_{R}(A): V_{R}(P)\right]_{r} \leqq[P: A]_{l}$ for any simple subring $A$ of $P$ such that $1 \in A$ and $[P: A]_{l}<\infty$. A similar result holds for any simple subring $B$ of $Q$ such that $1 \in B$ and $[Q: B]_{l}<\infty$.

Proof. Let $M$ be a minimal submodule of the $P_{l}-R_{r}$-module $R$. Then $R=P Q M R=Q M=\sum_{x \in Q} x M$, where $x M$ is 0 or $P_{l}-R_{r}$-isomorphic to $M$, that is, $x M$ is 0 or $P_{l}-R_{r}$-irreducible. The assertion of (ii) is a direct consequence of (i). Set $V_{R}(P)=\sum F f_{i j}$, where the $f_{i j}$ 's are matrix units and the centralizer $F$ of $\left\{f_{i j}\right\}$ in $V_{R}(P)$ is a division ring. Set $P^{\prime}=\sum P f_{i j}$. Then, noting here $P^{\prime} F=P V_{R}(P) \supset P Q, R$ is $P_{l}^{\prime} F_{l}-R_{r}$-irreducible. Since $V_{R}\left(P^{\prime}\right)$ $=F$ (division ring), $R$ is $P_{l}^{\prime}$ - $R_{r}$-irreducible. Clearly, for nonzero $v$ of $R$, 
$\left(v_{l} \mid P^{\prime}\right) R_{r}$ is (left) $P_{r}^{\prime}$-(right) $R_{r}$-irreducible in $\operatorname{Hom}\left(P^{\prime}, R\right)$, and $x \rightarrow\left(v_{l} \mid P^{\prime}\right) x_{r}$ is a module-isomorphism of $R$ onto $\left(v_{l} \mid P^{\prime}\right) R_{r}$. Set $A^{\prime}=V_{R}(A)$. Then we have $\left(A_{l}^{\prime} \mid P^{\prime}\right) R_{r} \subset \operatorname{Hom}_{A_{l}}\left(P^{\prime}, R\right)$ and

$$
\left[\left(A_{l}^{\prime} \mid P^{\prime}\right) R_{r}: R_{r}\right]_{r} \leqq\left[\operatorname{Hom}_{A_{l}}\left(P^{\prime}, R\right): R_{r}\right]_{r}=\left[P^{\prime}: A\right]_{l}=\left[P^{\prime}: P\right]_{l}[P: A]_{l} \text {. }
$$

Since $\left[A^{\prime}: F\right]_{r}=\left[A^{\prime}: V_{R}(P)\right]_{r}\left[V_{R}(P): F\right]_{r}$ and $\left[V_{R}(P): F\right] r=\left[P^{\prime}: P\right]_{l}$, it suffices to prove that $\left[A^{\prime}: F\right]_{r}=\left[\left(A_{l}^{\prime} \mid P^{\prime}\right) R_{r}: R_{r}\right]_{r}$. Hence, we shall show that for any subset $W$ of $A^{\prime}, W_{l} \mid P^{\prime}$ is linearly dependent over $R_{r}$ if and only if $W$ is linearly right-dependent over $F$. Assume that

$$
\sum v_{i} x_{i}=0 \quad\left(v_{i} \in W, x_{i} \in F\right)
$$

is a nontrivial relation. Then, for any $y \in P^{\prime}$, we have

$$
0=\left(\sum v_{i} x_{i}\right) y=\sum v_{i} y x_{i}=y\left(\sum\left(v_{i l} \mid P^{\prime}\right) x_{i r}\right) .
$$

This implies that $W_{l} \mid P^{\prime}$ is linearly dependent over $R_{r}$. Conversely, assume that

$$
\sum_{1}^{n}\left(v_{i l} \mid P^{\prime}\right) x_{i r}=0 \quad\left(v_{i} \in W, x_{i} \in R\right)
$$

is a nontrivial relation of shortest length. Since $\left(v_{i l} \mid P^{\prime}\right) R_{r}$ is (left) $P_{r}^{\prime}$ (right) $R_{r}$-irreducible, without loss of generality, we may assume that $x_{1}$ $=-1 ;\left(v_{1 l} \mid P^{\prime}\right)=\sum_{2}^{n}\left(v_{i l} \mid P^{\prime}\right) x_{i r}$. Then, for any $y \in P^{\prime}$, we have

$$
0=y_{r}\left(v_{1 l} \mid P^{\prime}\right)-\left(v_{1 l} \mid P^{\prime}\right) y_{r}=\sum_{2}^{n}\left(v_{i l} \mid P^{\prime}\right)\left(y x_{i}-x_{i} y\right) r .
$$

This implies that $\left(y x_{i}-x_{i} y\right)_{r}=0$ and so $y x_{i}=x_{i} y$ for any $y \in P^{\prime}$. Hence $x_{i} \in F(i=1, \cdots, n)$. We have therefore a nontrivial relation $\sum_{1}^{n} v_{i} x_{i}=0$ $\left(x_{i} \in F\right)$.

Corollary 1. Let $P$ be a subring of $R$ containing 1 such that $R$ is $P_{l^{-}} R_{r^{-}}$ irreducible. Then $\left[V_{R}(A): V_{R}(P)\right]_{r} \leqq[P: A]_{l}$ for any simple subring $A$ of $P$ such that $1 \in A$ and $[P: A]_{l}<\infty$.

Corollary 2. Let $S$ be simple and $R$ left locally finite over $S$. Let $T$ be a subring of $R$ containing $S$ such that $\left\{e_{i j}\right\} \subset T$ and $[T: S]_{l}<\infty$. Then $\left[V: V_{R}(T)\right]_{r} \leqq[T: S]_{l}$ and $\left[V: V_{R}(T)\right]_{l} \leqq[T: S]_{l}$. In particular, if $H \cap T$ is simple, then $\left[V: V_{R}(T)\right]_{r} \leqq[T: H \cap T]_{l}$ and $\left[V: V_{R}(T)\right]_{l} \leqq[T: H \cap T]_{l}$.

Proof. For an arbitrary finite subset $F$ of $V$, we set $T^{\prime}=T[F]$. Then $T^{\prime}$ is $T_{l}-T_{r}^{\prime}$-irreducible and $T_{l}^{\prime}$ - $T_{r}^{\prime}$-irreducible. Hence, by Corollary 1 , we have $\left[V_{T^{r}}(S): V_{T}(T)\right]_{r} \leqq[T: S]_{l}$ and $\left[V_{T^{r}}(S): V_{T^{\prime}}\left(T^{\prime}\right)\right]_{r} \leqq\left[T^{\prime}: S\right]_{l}$. Since

$$
\left[V_{T^{v}}(S): V_{T}\left(T^{\prime}\right)\right]_{r}=\left[V_{T^{v}}(S): V_{T^{v}}\left(T^{\prime}\right)\right]_{l}
$$

and 


$$
\left[V_{T^{r}}(T): V_{T^{(}}\left(T^{\prime}\right)\right]_{r}=\left[V_{T^{v}}(T): V_{T^{\prime}}\left(T^{\prime}\right)\right]_{l}
$$

it follows that $\left[V_{T}(S): V_{T}(T)\right]_{l}=\left[V_{T}(S): V_{T^{T}}(T)\right]_{r} \leqq[T: S]_{l}$. Noting here $V_{T}(S) \subset V$ and $V_{T}(T) \subset V_{R}(T)$, we obtain our assertion.

If two subrings $T, S^{\prime}$ of $R$ satisfy the following conditions, then we say that $S^{\prime}$ is left linearly disjoint from $T: S^{\prime}$ contains a linearly independent left basis over $T \cap S^{\prime}$ and for any linearly independent left basis $\left\{d_{i} ; i \in I\right\}$ of $R$ over $T \cap S^{\prime}$, the sum $\sum_{i \in I} T d_{i}$ is direct and $T d_{i}$ is (left) $T$-isomorphic to $T$. If $S^{\prime}$ is left linearly disjoint and right linearly disjoint from $T$, then we say that $S^{\prime}$ is linearly disjoint from $T$. The next lemma is partially similar to [4, Lemma 3.2$]$.

Lemma 2. Let $H$ be simple, and let $S^{\prime}$ be a subring of $R$ containing $S\left[\left\{d_{h k}\right\}\right]$ such that $\left.J(\circlearrowleft)\left(S^{\prime}, R\right), R\right)=S^{\prime}$. Then $H \cap S^{\prime}$ is simple and $S^{\prime}$ is linearly disjoint from $H$.

Proof. Set $H^{\prime}=H \cap S^{\prime}$. Then, noting that $H^{\prime} \supset\left\{d_{h k}\right\}$ and $x^{-1} \in S^{\prime}$ for any regular $x \in S^{\prime}, V_{H^{\prime}}\left(\left\{d_{h k}\right\}\right)$ is a division ring, and so $H^{\prime}$ is simple. Now, choose a linearly independent left basis $\left\{d_{i} ; i \in I\right\}$ of $S^{\prime}$ over $H^{\prime}$. Then we have

$$
\sum_{i} H d_{i}=\sum_{i, h, k} K d_{h k} d_{i} \quad(K \text { is a division ring }) .
$$

Hence, it will suffice to prove that $\left\{d_{h k} d_{i}\right\}$ is linearly left-independent over $K$. If not, without loss of generality, we may assume that

$$
d_{11} d_{1}=\sum a_{h k i} d_{h k} d_{i} \quad\left(a_{h k i} \in K\right)
$$

is a nontrivial relation of the shortest length. Then, as there exists some $a_{h^{\prime} k^{\prime} i^{\prime}}$ not contained in $K \cap S^{\prime}$, we can find some $\sigma \in(S)\left(S^{\prime}, R\right)$ such that $a_{h^{\prime} k^{\prime} i^{\prime}} \neq a_{h^{\prime} k^{\prime} i^{\prime}} \sigma(\in K)$. Hence, we have a nontrivial relation of shorter length:

$$
0=d_{11} d_{1}-\left(d_{11} d_{1}\right) \sigma=\sum\left(a_{h k i}-a_{h k i} \sigma\right) d_{h k} d_{i} .
$$

This contradiction implies that the $d_{h k} d_{i}$ 's are linearly left-independent over $K$. Moreover, if we use similar method for a linearly independent right basis $\left\{d_{j}^{*} ; j \in J\right\}$ of $S^{\prime}$ over $H^{\prime}$, then the $d_{j} d_{h k}$ 's are linearly rightindependent over $K$.

Corollary 3. Let $R$ be Galois and left locally finite over $S$, and $H$ simple. Let $T$ be a subring of $R$ containing $S\left[\left\{e_{i j}\right\},\left\{d_{h k}\right\}\right]$ with $[T: S]_{l}<\infty$. Set $T^{\prime}=J(\$(T, R), R)$. Then we have:

(i) $\left[H \cap T^{\prime}: S\right]<\infty$ and $T^{\prime}$ is linearly disjoint from $H$, and

(ii) $H_{\lambda} T^{\prime}$ is a simple ring for any subring $H_{\lambda}$ of $H$ containing $H \cap T^{\prime}$. In particular, $H T^{\prime}$ is a simple ring.

Proof. Set $H^{\prime}=H \cap T^{\prime}$. Then $T^{\prime}$ is linearly disjoint from $H$ by Lemma 
2. Further, $H^{\prime}$ is finite over $S$ by Proposition $\gamma(\mathrm{i})$. Let $T_{i}$ be an arbitrary subring of $T^{\prime}$ containing $H^{\prime}[T]$ with $\left[T_{i}: H^{\prime}\right]_{l}<\infty$. For any element $a$ of $H$, we set $N=H^{\prime}\left[\left\{a_{\sigma}, \sigma \in\left(\$\left(T^{\prime}, R\right)\right\}\right]\right.$, and

$$
T_{i}^{\prime}=J\left(\Theta\left(T^{\prime}, R\right) \mid T_{i}[N], T_{i}[N]\right) .
$$

Then $N$ and $T_{i}[N]$ are outer Galois over $H^{\prime}$ and $T_{i}^{\prime}$, respectively; the Galois groups of these are $\$\left(T^{\prime}, R\right) \mid N$ and $\$\left(T^{\prime}, R\right) \mid T_{i}[N]$, respectively. Hence, by Proposition $\beta$, we have $\left[H^{\prime}[a]: H^{\prime}\right]=\#\left(\$\left(T^{\prime}, R\right) \mid H^{\prime}[a]\right)$ $=\#\left(\$\left(T^{\prime}, R\right) \mid T_{i}^{\prime}[a]\right)=\left[T_{i}^{\prime}[a]: T_{i}^{\prime}\right]$. Since $T^{\prime}$ is linearly disjoint from $H$ over $H^{\prime}$ and $H^{\prime} \subset T_{i}^{\prime} \subset T^{\prime}$, it follows that

$$
\begin{aligned}
{\left[H^{\prime}[a] T_{i}^{\prime}: H^{\prime}\right]_{l} } & =\left[H^{\prime}[a] T_{i}^{\prime}: H^{\prime}[a]\right]_{l}\left[H^{\prime}[a]: H^{\prime}\right]_{l} \\
& =\left[T_{i}^{\prime}: H^{\prime}\right]_{l}\left[T_{i}^{\prime}[a]: T_{i}^{\prime}\right]_{l}=\left[T_{i}^{\prime}[a]: H^{\prime}\right]_{l} .
\end{aligned}
$$

This implies that $H^{\prime}[a] T_{i}^{\prime}=T_{i}^{\prime}[a] \supset T_{i}[a]$. For an arbitrary subring $H_{i}$ of $H_{\lambda}$ containing $H^{\prime}$ with $\left[H_{i}: H^{\prime}\right]<\infty, H_{i}=H^{\prime}[a]$ for some $a \in H_{i}$ by Proposition $\beta$. Hence, $T^{\prime}\left[H_{\lambda}\right]=\bigcup_{a \in H_{\lambda}, i} T_{i}[a]=\bigcup H^{\prime}[a] T_{i}^{\prime}=H_{\lambda} T^{\prime}$. Noting that $T^{\prime}\left[H_{\lambda}\right] \supset\left\{e_{i j}\right\}$ and $T^{\prime}\left[H_{\lambda}\right]$ is left locally finite over $S, H_{\lambda} T^{\prime}$ is a simple ring.

Corollary 4. Let $R$ be Galois and left locally finite over $S$, and $H$ simple. If $R$ is Galois over every regular subring $S^{\prime}$ of $T$ containing $S$ with $\left[S^{\prime}: S\right]_{l}$ $<\infty$, then $R$ is left locally finite over any subring $H_{\lambda}$ of $H$ containing $S$.

Proof. For any finite subset $F$ of $R$, we set $T=S\left[\left\{e_{i j}\right\},\left\{d_{h k}\right\}, F\right]$, and $H \cap T=H^{\prime}$. Since $R$ is Galois over $T, H_{\lambda}\left[H^{\prime}\right] T$ is a simple ring and $T$ is linearly disjoint from $H$ over $H^{\prime}$. Hence, by Proposition $\beta$, we have

$$
\begin{aligned}
{\left[H_{\lambda}[F]: H_{\lambda}\right]_{l} } & \leqq\left[H_{\lambda}\left[H^{\prime}\right] T: H_{\lambda}\right]_{l}=\left[H_{\lambda}\left[H^{\prime}\right] T: H_{\lambda}\left[H^{\prime}\right]\right]_{l}\left[H_{\lambda}\left[H^{\prime}\right]: H_{\lambda}\right]_{l} \\
& =\left[T: H^{\prime}\right]_{l}\left[H_{\lambda}\left[H^{\prime}\right]: H_{\lambda}\right]_{l}<\infty .
\end{aligned}
$$

Proposition 1. $\left(\mathrm{A}_{l}\right) \Longrightarrow\left(\mathrm{B}_{l}\right) \Longrightarrow\left(\mathrm{A}_{r}\right) \Longrightarrow\left(\mathrm{B}_{r}\right) \Longrightarrow\left(\mathrm{A}_{l}\right)$. If one of these is fulfilled, then $R$ is two-sided locally finite over $S$ and over $H$.

Proof. $\left(\mathrm{A}_{l}\right) \Longrightarrow\left(\mathrm{B}_{l}\right)$ is a direct consequence of Proposition $\alpha(\mathrm{i}, \mathrm{iii})$. Let $R$ satisfy $\left(\mathrm{B}_{l}\right)$ over $S$. Since $R$ is $S_{l} V_{l}-R_{r}$-irreducible, $H\left(=V_{R}(S)\right)$ is simple by Lemma 1(ii). For any finite subset $F$ of $R$, we set

$$
\left.T=S\left[\left\{e_{i j}\right\},\left\{d_{h k}\right\}, F\right], \quad T^{\prime}=J(\$)(T, R), R\right), \quad \text { and } \quad H^{\prime}=H \cap T^{\prime} .
$$

Then $\left[V: V_{R}(T)\right]_{l} \leqq[T: S]_{l}$ by Corollary 2 , and $V_{R}(T)=V_{R}\left(T^{\prime}\right)$, and whence $\left[V: V_{R}\left(T^{\prime}\right)\right]_{l}<\infty$. Hence, by Lemma 1 and Lemma 2, we have

$$
\left[T^{\prime}: H^{\prime}\right]_{r}=\left[T^{\prime} H: H\right]_{r} \leqq\left[V_{R}^{2}\left(T^{\prime}\right): H\right]_{r} \leqq\left[V: V_{R}\left(T^{\prime}\right)\right]_{l}<\infty .
$$

On the other hand, noting that $R$ is $T_{r}^{\prime}$ - $R_{l}$-irreducible, it follows that

$$
\left[T^{\prime}: H^{\prime}\right]_{r} \geqq\left[V: V_{R}\left(T^{\prime}\right)\right]_{l},
$$

by the proposition symmetric to Corollary 1. Combining (1) and (2), we 
have $\left[T^{\prime}: H^{\prime}\right]_{r}=\left[V: V_{R}\left(T^{\prime}\right)\right]_{l}$. Hence, we have $\operatorname{Hom}_{S_{r}}\left(T^{\prime}, R\right)=\left(\Theta \mid T^{\prime}\right) R_{l}$ by the proposition symmetric to Proposition $\gamma\left(\right.$ ii). Since $T^{\prime} \supset F$ and (1), it follows that $\left(\mathrm{B}_{l}\right) \Longrightarrow\left(\mathrm{A}_{r}\right)$, and $R$ is right locally finite over $S$ and over $H$. $\left(A_{r}\right) \Longrightarrow\left(B_{r}\right) \Longrightarrow\left(A_{l}\right)$ is the proposition symmetric to $\left(A_{l}\right) \Longrightarrow\left(B_{l}\right)$ $\Longrightarrow\left(\mathrm{A}_{r}\right)$, and so, $T$ is left locally finite (over $S$ and) over $H$.

Proposition 2. $\left(\mathrm{C}_{l}\right) \Longrightarrow\left(\mathrm{A}_{l}\right),\left(\mathrm{D}_{l}\right) \Longrightarrow\left(\mathrm{A}_{l}\right),\left(\mathrm{E}_{l}\right) \Longrightarrow\left(\mathrm{A}_{l}\right)$, and $\left(\mathrm{E}_{l}^{*}\right)$ $\Longrightarrow\left(A_{l}\right)$. Symmetrically, $\left(C_{r}\right) \Longrightarrow\left(A_{r}\right), \quad\left(D_{r}\right) \Longrightarrow\left(A_{r}\right), \quad\left(E_{r}\right) \Longrightarrow\left(A_{r}\right)$, and $\left(\mathrm{E}_{r}^{*}\right) \Longrightarrow\left(\mathrm{A}_{r}\right)$.

Proof. In any case of the conditions $\left(\mathrm{C}_{l}\right),\left(\mathrm{D}_{l}\right),\left(\mathrm{E}_{l}\right)$, and $\left(\mathrm{E}_{l}^{*}\right)$, it is clear that $R$ is Galois and left locally finite over $S$, and $H$ is simple (in case $\left(\mathrm{C}_{l}\right)$, $H$ is simple by Lemma 1). For any arbitrary finite subset $F$ of $R$, we set $T=S\left[\left\{e_{i j}\right\},\left\{d_{h k}\right\}, F\right], \quad T^{\prime}=J(\$(T, R), R)$, and $H^{\prime}=H \cap T^{\prime}$. Then, by Corollary 3 , we have

$$
\left[H^{\prime}: S\right]<\infty .
$$

Since $R$ is $H^{\prime}[T]_{l}-R_{r}$-irreducible and $\left[H^{\prime}[T]: H^{\prime}\right]_{l}<\infty$, we have $\left[T^{\prime}: H^{\prime}\right]_{l}$ $\geqq\left[H^{\prime}[T]: H^{\prime}\right]_{l} \geqq\left[V_{R}\left(H^{\prime}\right): V_{R}\left(H^{\prime}[T]\right)\right]_{r}=\left[V: V_{R}(T)\right]_{r}=\left[V: V_{R}\left(T^{\prime}\right)\right]_{r}$ by Lemma 1. This inequality and Corollary 3 imply

$$
\left[V_{R}^{2}\left(T^{\prime}\right): H\right]_{l} \geqq\left[H T^{\prime}: H\right]_{l}=\left[T^{\prime}: H^{\prime}\right]_{l} \geqq\left[V: V_{R}\left(T^{\prime}\right)\right]_{r},
$$

where $\left[V: V_{R}\left(T^{\prime}\right)\right]_{r}<\infty$. Finally, we shall show that

$$
\left[T^{\prime}: H^{\prime}\right]_{l}=\left[V: V_{R}\left(T^{\prime}\right)\right]_{r}
$$

(and then $\left[T^{\prime}: S\right]_{l}<\infty$ by (1) and (2)), and this and Proposition $\gamma($ (ii) will imply $\operatorname{Hom}_{S_{l}}\left(T^{\prime}, R\right)=\left(\mathbb{S} \mid T^{\prime}\right) R_{r}$, which is the desired one. In case $\left(\mathrm{C}_{l}\right)$, we have $\left[V_{R}^{2}\left(T^{\prime}\right): H\right]_{l} \leqq\left[V: V_{R}\left(T^{\prime}\right)\right]_{r}$ by the proposition symmetric to Corollary 1. Hence, $\left[T^{\prime}: H^{\prime}\right]_{l}=\left[V: V_{R}\left(T^{\prime}\right)\right]_{r}$ by (2). In case $\left(D_{l}\right)$,

$$
\left[V_{R}^{2}\left(T^{\prime}\right): H\right]_{l}=\left[V_{R}^{2}(T): H\right]_{l}=\left[V: V_{R}(T)\right]_{r}=\left[V: V_{R}\left(T^{\prime}\right)\right]_{r} .
$$

Hence, $\left[T^{\prime}: H^{\prime}\right]_{l}=\left[V: V_{R}\left(T^{\prime}\right)\right]_{r}$. In case $\left(\mathrm{E}_{l}\right)$, we have $T^{\prime}=T$, and so, $H T^{\prime}$ is a regular subring with $\left[H T^{\prime}: H\right]_{l}<\infty$ by Corollary 3 . Hence, $\left[H T^{\prime}: H\right]_{l}=\left[V: V_{R}\left(H T^{\prime}\right)\right]_{r}=\left[V: V_{R}\left(T^{\prime}\right)\right]_{r}$. This implies that $\left[T^{\prime}: H^{\prime}\right]_{l}$ $=\left[V: V_{R}\left(T^{\prime}\right)\right]_{r}$. Case $\left(\mathrm{E}_{l}^{*}\right)$ is contained in $\left(\mathrm{E}_{l}\right)$.

Proposition 3. $\left(\mathrm{A}_{l}\right) \Longrightarrow\left(\mathrm{C}_{l}\right),\left(\mathrm{D}_{l}\right),\left(\mathrm{E}_{l}\right)$, and $\left(\mathrm{E}_{l}^{*}\right)$. Symmetrically, $\left(\mathrm{A}_{r}\right)$ $\Longrightarrow\left(\mathrm{C}_{r}\right)$, $\left(\mathrm{D}_{r}\right),\left(\mathrm{E}_{r}\right)$, and $\left(\mathrm{E}_{r}^{*}\right)$. If $R$ satisfies $\left(\mathrm{A}_{l}\right)$ over $S$, then, for every regular subring $S^{\prime}$ of $R$ containing $S$ with $\left[S^{\prime}: S\right]_{l}<\infty, R$ satisfies $\left(\mathrm{A}_{l}\right)$ over $S^{\prime}$, and $\left[S^{\prime}: S\right]_{l}=\left[S^{\prime}: S\right]_{r}$.

Proof. Let $R$ satisfy $\left(\mathrm{A}_{l}\right)$ over $S$, and let $S^{\prime}$ be as in our Proposition 3 . Then, by Proposition $\alpha\left(\mathrm{i}\right.$, iii) and Lemma $1, R$ is Galois over $S^{\prime}$ and $S_{l}^{\prime} V_{R}\left(S^{\prime}\right)_{l}-R_{r}$-irreducible, and $V_{R}^{2}\left(S^{\prime}\right)$ is simple. In particular, $R$ is Galois over $S$ and $S_{l} V_{l}-R_{r}$-irreducible, and $H$ is simple. Evidently, $R$ satisfies 
$\left(\mathrm{B}_{l}\right)$ over $S^{\prime}$ and, hence, satisfies $\left(\mathrm{A}_{l}\right)$ over $S^{\prime}$ by Proposition 1. Moreover, $\left(A_{l}\right) \Longrightarrow\left(C_{l}\right)$ is a direct consequence of $\left(A_{l}\right) \Longrightarrow\left(B_{r}\right) \Longrightarrow\left(C_{l}\right)$.

Now, we set $T=S^{\prime}\left[\left\{e_{i j}\right\},\left\{d_{h k}\right\},\left\{f_{w}\right\}\right]$, where the $f_{w}$ 's are matrix units of $V_{R}^{2}\left(S^{\prime}\right)$ (simple ring) such that the centralizer of $\left\{f_{w}\right\}$ in $V_{R}\left(S^{\prime}\right)$ is a division ring. Set $H^{\prime}=H \cap T$. Then, by Corollary 2 and Lemma 1(iii) and Lemma 2, we have

$$
\left[T: H^{\prime}\right]_{l} \geqq\left[V: V_{R}(T)\right]_{l} \geqq\left[V_{R}^{2}(T): H\right]_{r} \geqq[T H: H]_{r}=\left[T: H^{\prime}\right]_{r} .
$$

Similarly $\left(\left(A_{l}\right) \Longleftrightarrow\left(A_{r}\right)\right)$, we have

$$
\left[T: H^{\prime}\right]_{r} \geqq\left[V: V_{R}(T)\right]_{r} \geqq\left[V_{R}^{2}(T): H\right]_{l} \geqq[H T: H]_{l}=\left[T: H^{\prime}\right]_{l} .
$$

Combining (1), (2) and $\left[H^{\prime}: S\right]_{l}=\left[H^{\prime}: S\right]_{r}$ (Proposition $\beta$ ), it follows that

$$
[T: S]_{l}=[T: S]_{r} \text { and }\left[V_{R}^{2}(T): H\right]_{l}=\left[V: V_{R}(T)\right]_{r} .
$$

Since $R$ satisfies $\left(\mathrm{A}_{l}\right)$ over $S^{\prime}$, there holds a similar result:

$$
\left[T: S^{\prime}\right]_{l}=\left[T: S^{\prime}\right]_{r} \text { and }\left[V_{R}^{2}(T): V_{R}^{2}\left(S^{\prime}\right)\right]_{l}=\left[V_{R}\left(S^{\prime}\right): V_{R}(T)\right]_{r} .
$$

Hence, by (3) and (4), we obtain that $\left[S^{\prime}: S\right]_{l}=\left[S^{\prime}: S\right]_{r}$ and $\left[V_{R}^{2}\left(S^{\prime}\right): H\right]_{l}$ $=\left[V: V_{R}\left(S^{\prime}\right)\right]_{r}$. This implies $\left(\mathrm{A}_{l}\right) \Longrightarrow\left(\mathrm{D}_{l}\right)$.

Finally, we shall show $\left(\mathrm{A}_{l}\right) \Longrightarrow\left(\mathrm{E}_{l}\right)$ and $\left(\mathrm{E}_{l}^{*}\right)$. Let $R^{\prime}$ be a regular subring of $R$ containing $H$ with $\left[R^{\prime}: H\right]_{l}<\infty$. Then there exists a regular subring $S^{\prime}$ of $R^{\prime}$ containing $S$ with $\left[S^{\prime}: S\right]_{l}<\infty$ such that $V_{R}\left(R^{\prime}\right)=V_{R}\left(S^{\prime}\right)$ (note that $\left.\left[R^{\prime}: H\right]_{l}<\infty\right)$. Since $\left(\mathrm{A}_{l}\right) \Longrightarrow\left(\mathrm{D}_{l}\right)$, it follows that $\left[V_{R}^{2}\left(S^{\prime}\right): H\right]_{l}$ $=\left[V: V_{R}\left(S^{\prime}\right)\right]_{r}$, and so, $\left[V_{R}^{2}\left(R^{\prime}\right): H\right]_{l}=\left[V: V_{R}\left(R^{\prime}\right)\right]_{r}$. Moreover, $R$ is $S_{l}^{\prime} V_{R}\left(S^{\prime}\right)_{l}$ - $R_{r}$-irreducible, that is, $R$ is $R_{l}^{\prime} V_{R}\left(R^{\prime}\right)_{l}-R_{r}$-irreducible. Hence $\left[R^{\prime}: H\right]_{l} \geqq\left[V: V_{R}\left(R^{\prime}\right)\right]_{r}$ by Lemma 1 (iii). Noting here $V_{R}^{2}\left(R^{\prime}\right) \supset R^{\prime} \supset H$, we have $R^{\prime}=V_{R}^{2}\left(R^{\prime}\right)$ and $\left[R^{\prime}: H\right]_{l}=\left[V: V_{R}\left(R^{\prime}\right)\right]_{r}$.

THEOREM 1. The conditions $\left(\mathrm{A}_{l}\right)-\left(\mathrm{E}_{r}^{*}\right)$ are all equivalent. If $R$ satisfies one of these over $S$, then $R$ satisfies these over $H$, and, for every regular subring $S^{\prime}$ of $R$ containing $S$ with $\left[S^{\prime}: S\right]_{l}<\infty, R$ satisfies these over $S^{\prime}$, and

$$
\begin{aligned}
{\left[S^{\prime}: S\right]_{l} } & =\left[S^{\prime}: S\right]_{r} \geqq\left[V: V_{R}\left(S^{\prime}\right)\right]_{l}=\left[V: V_{R}\left(S^{\prime}\right)\right]_{r}=\left[V_{R}^{2}\left(S^{\prime}\right): H\right]_{l} \\
& =\left[V_{R}^{2}\left(S^{\prime}\right): H\right]_{r}=\left[S^{\prime}: H \cap S^{\prime}\right]_{l}=\left[S^{\prime}: H \cap S^{\prime}\right]_{r} .
\end{aligned}
$$

Proof. The first assertion is a combination of Propositions 1,2 and 3. Evidently, $R$ satisfies $\left(\mathrm{E}_{l}\right)$ over $H$. Let $S^{\prime}$ be as in our theorem. Then, $R$ is $S_{l}^{\prime} V_{R}\left(S^{\prime}\right)_{l}-R_{r}$-irreducible and $S_{r}^{\prime} V_{R}\left(S^{\prime}\right)_{r}-R_{l}$-irreducible. Hence, we have $\left[S^{\prime}: S\right]_{l} \geqq\left[V: V_{R}\left(S^{\prime}\right)\right]_{r}$ and $\left[S^{\prime}: S\right]_{r} \geqq\left[V: V_{R}\left(S^{\prime}\right)\right]_{l}$ (Lemma 1(iii)). Then, by $\left(D_{l}\right),\left(D_{r}\right)$ and Proposition 3 (over $S$ and over $H$ ), we obtain our last assertion except for the equalities $\left[V_{R}^{2}\left(S^{\prime}\right): H\right]_{r}=\left[S^{\prime}: H \cap S^{\prime}\right]_{l}=\left[S^{\prime}: H \cap S^{\prime}\right]_{r}$. To prove this, it suffices to show that $\left[S^{\prime}: H \cap S^{\prime}\right]_{l}=\left[V_{R}^{2}\left(S^{\prime}\right): H\right]_{l}$. Since $R$ satisfies $\left(\mathrm{A}_{l}\right)$ over $H \cap S^{\prime},\left[S^{\prime}: H \cap S^{\prime}\right]_{l} \geqq\left[V_{R}^{2}\left(S^{\prime}\right): H\right]_{l}$. Hence, we shall only show that $\left[S^{\prime}: H \cap S^{\prime}\right]_{l} \leqq\left[V_{R}^{2}\left(S^{\prime}\right): H\right]_{l}$. Let $H^{*}$ be a subring of $H$ 
containing $\left(H \cap S^{\prime}\right)\left[\left\{d_{h k}\right\}\right]$ with $\left[H^{*}: S\right]<\infty$. Set $S^{*}=S^{\prime}\left[H^{*}\right]$. Then $S^{\prime} \subset S^{*} \subset V_{R}^{2}\left(S^{\prime}\right)$. Noting that $V_{R}^{2}\left(S^{\prime}\right)$ is outer Galois and locally finite over $S^{\prime}, S^{*}$ is simple by Proposition $\beta$, that is, $S^{*}$ is a regular subring finite over $S$, and whence, $R$ is Galois over $S^{*}$. Hence, by Lemma 2, $\left[S^{*}: H \cap S^{*}\right]_{l}$ $=\left[H S^{*}: H\right]_{l} \leqq\left[V_{R}^{2}\left(S^{\prime}\right): H\right]_{l}$. Moreover, by Proposition $\beta$ and its remark,

$$
\begin{aligned}
{\left[S^{*}: S^{\prime}\right] } & \left.=\#(\$)\left(S^{\prime}, R\right) \mid S^{*}\right)=\#\left(\$\left(S^{\prime}, R\right) \mid H^{*}\right) \\
& =\#\left(\$\left(S^{\prime}, R\right) \mid H \cap S^{*}\right)=\left[H \cap S^{*}: H \cap S^{\prime}\right] .
\end{aligned}
$$

We have, therefore, $\left[S^{\prime}: H \cap S^{\prime}\right]_{l}=\left[S^{*}: H \cap S^{*}\right]_{l} \leqq\left[V_{R}^{2}\left(S^{\prime}\right): H\right]_{l}$.

REMARK 1 . Let $R$ satisfy $\left(\mathrm{A}_{l}\right)$ over $S$. For any finite subset $F$, set $T=S\left[\left\{e_{i j}\right\}, F\right]$. Then, $T$ is a regular subring finite over $S$. Hence, $[T: T \cap H]$ $=\left[V: V_{R}(T)\right]$. Further, $R$ is $T_{l}-R_{r}$-irreducible. Hence, if $S^{*}$ is a subgroup of $(\$)$ such that $S^{*} \supset\langle V\rangle$ and $J\left(S^{*}, R\right)=S$, then $(\$) T=S^{*} \mid T$ (Proposition $\gamma(\mathrm{ii}))$ and so $\left(\mathscr{S} \mid F=\left(\mathfrak{S}^{*} \mid F\right.\right.$. This result is contained in [4], which will be required later.

REMARK 2. Let $R$ be Galois and left locally finite over $S$. If $R$ is a division ring, then $R$ satisfies $\left(\mathrm{A}_{l}\right)$ over $S$, which is a direct consequence of Jacobson's density theorem. On the other hand, this result is a direct consequence of the equivalence $\left(B_{l}\right) \Longleftrightarrow\left(A_{l}\right)$.

Remark 3. Let $R$ be Galois and left locally finite over $S$. If $V$ is finite over the center $C_{0}$ of $V$, then $R$ satisfies $\left(\mathrm{A}_{l}\right)$ over $S$ [4, Theorems 2.2, 2.4], and this proof is very complicated. However, this theorem is a consequence of the equivalence $\left(D_{l}\right) \Longleftrightarrow\left(A_{l}\right)$. We shall show that $R$ satisfies $\left(D_{l}\right)$ over $S$. Let $S^{\prime}$ be a regular subring of $R$ containing $S$ with $\left[S^{\prime}: S\right]_{l}<\infty$. Set $T=S^{\prime}\left[\left\{e_{i j}\right\},\left\{d_{1}, \cdots, d_{n}\right\}\right]$, where $\left\{d_{i}\right\}$ is a $C_{0}$-base of $V$. Then $\left[V: V_{R}(T)\right]_{r}$ $\leqq[T: S]_{l}<\infty$ (Lemma 1), $V_{R}(T) \subset C_{0} \subset V, V_{R}(T) \subset V_{R}\left(S^{\prime}\right) \subset V \subset V_{R}^{2}(T)$, and $\left\{e_{i j}\right\} \subset V_{R}^{2}(T)$. Hence, $V_{R}(T)$ is the center of the simple ring $V_{R}^{2}(T)$. Since $\left[V: V_{R}(T)\right]<\infty, V_{R}(V)=H$ and $V_{R}^{2}\left(S^{\prime}\right)$ are both simple (the fundamental theorem of simple rings). We have then $\left[V_{R}^{2}(T): V_{R}(V)\right]=\left[V: V_{R}(T)\right]$ and $\left[V_{R}^{2}(T): V_{E}^{2}\left(S^{\prime}\right)\right]=\left[V_{R}\left(S^{\prime}\right): V_{R}(T)\right]$. From this, it follows that $\left[V_{R}^{2}\left(S^{\prime}\right): H\right]=\left[V: V_{R}\left(S^{\prime}\right)\right]$.

REMARK 4. If, for any finite subset $F$ of $R$, there exists a subring of $R$ containing $F$ which is Galois and finite over $S$, then we say that $R$ is locally Galois over $S$. If $R$ is Galois and locally Galois over $S$, then $R$ satisfies $\left(\mathrm{A}_{l}\right)$ over $S$, and this proof has been given in [4, Theorems 2.2, 2.3]. On the other hand, this is an easy consequence of the equivalence $\left(B_{l}\right) \Longleftrightarrow$ $\left(\mathrm{A}_{l}\right)$. We shall show that $R$ satisfies $\left(\mathrm{B}_{l}\right)$ over $S$. Let $a$ be a nonzero element of $R$, and $x$ any element of $R$. Then, there exists a subring $N$ of $R$ containing $\{a, x\}$ which is Galois and finite over $S$. Since $N$ satisfies $\left(D_{l}\right)$ over $S$ (the fundamental theorem of simple rings), $N$ is $S_{l} V_{N}(S)_{l}-N_{r^{-}}$ irreducible. Hence, $N=S V_{N}(S) a N \ni x$. This implies that $R=S V_{R}(S) a R$, that is, $R$ is $S_{l} V_{l}-R_{r}$-irreducible. 
2. On $\$$. This section is about the regular subgroups of $\mathbb{H}$. Now, we shall recall the definitions of regular subgroups of $(\xi)$ as in [4]. Let $R$ be Galois over $S$, and let $\left(S^{\prime}\right.$ be a subgroup of $S^{\prime}$. Then $V_{R}\left(J\left(S^{\prime}, R\right)\right)$ will be denoted by $V\left(\xi^{\prime}\right)$. In our paper [4], $V\left(\left(^{\prime}\right)\right.$ was denoted by $V_{\leftrightarrow}$. Further, by $I\left(\$^{\prime}\right)$ we denote the subring of $R$ generated by the set of regular elements inducing inner automorphisms belonging to (S'). Clearly, $I\left(\mathbb{S}^{\prime}\right)$ $\subset V\left(\mathbb{S}^{\prime}\right) \subset\left(y^{\prime}\right.$.

(i) If $I\left(B^{\prime}\right)$ is simple and $\left\langle I\left(\mathbb{S}^{\prime}\right)\right\rangle \subset\left(\mathcal{S}^{\prime}\right.$, then $\mathcal{S}^{\prime}$ is said to be $N$-regular. If, in addition, $\left[V: I\left(\mathbb{S}^{\prime}\right)\right]_{r}<\infty$, then $\left(\xi^{\prime}\right.$ is said to be $N_{f}$-regular.

(ii) If $V\left(\mathbb{S}^{\prime}\right)$ is simple and $\left\langle V\left(\mathbb{S}^{\prime}\right)\right\rangle \subset\left(\mathbb{S}^{\prime}\right.$, then $\mathbb{S}^{\prime}$ is said to be (*)regular $\left(B^{\prime}\right)^{\prime}$ is (*)-regular if and only if $\left(S^{\prime}\right.$ is $N$-regular and $\left.I\left(\Theta^{\prime}\right)=V\left(\Theta^{\prime}\right)\right)$. If, in addition, $\left[V: V\left(\mathbb{S}^{\prime}\right)\right]_{r}<\infty$, then $\mathcal{S}^{\prime}$ is said to be $\left(*_{f}\right)$-regular.

(iii) If $\left(^{\prime}\right.$ is (*)-regular and $J\left(S^{\prime}, R\right)$ is simple, then $\left(^{\prime}\right.$ is said to be regular. If, in addition, $\left[V: V\left(\mathcal{S}^{\prime}\right)\right]_{r}<\infty$, then $\mathcal{S}^{\prime}$ is said to be f-regular. Similarly, a regular subring $S^{\prime}$ of $R$ containing $S$ is said to be $f$-regular provided that $\left[V: V_{R}\left(S^{\prime}\right)\right]_{r}<\infty$. If $S^{\prime}$ is a regular subring $S^{\prime}$ of $R$ finite over $S$, then $S^{\prime}$ is f-regular and $(S)\left(S^{\prime}, R\right)$ is f-regular (Theorem 1). In general, if $S^{\prime}$ is an $f$-regular subring of $R$, then $S^{\prime}\left(S^{\prime}, R\right)$ is $\left(*_{f}\right)$-regular. One will see later (Theorem 3$)$ that $(S)\left(S^{\prime}, R\right)$ is f-regular. On the other hand, if $\left(S^{\prime}\right.$ is f-regular then $J\left(\mathcal{S}^{\prime}, R\right)$ is f-regular.

At first, we shall prove the next lemma which will play important roles in our subsequent considerations.

Lemma 3. Let $R$ satisfy $\left(\mathrm{A}_{l}\right)$ over $S$. Then, for subrings $R^{\prime}, V^{\prime}$ of $R$ such that $R^{\prime} \supset H, V \supset V^{\prime}, V_{R}\left(R^{\prime}\right)=V^{\prime}$ and $V_{R}\left(V^{\prime}\right)=R^{\prime}$, the following hold:

(i) If $R^{\prime}$ is a simple ring with $\left[R^{\prime}: H\right]_{l}<\infty$, then $V^{\prime}$ is simple, and $\left[R^{\prime}: H\right]$ $=\left[V: V^{\prime}\right]$.

(ii) If $V^{\prime}$ is a simple ring with $\left[V: V^{\prime}\right]_{r}<\infty$, then $R^{\prime}$ is simple, and $\left[V: V^{\prime}\right]$ $=\left[R^{\prime}: H\right]$.

Proof. (i) Since $R$ satisfies $\left(\mathrm{A}_{l}\right)$ over $H$ (Theorem 1 ), $R$ is $R_{l}^{\prime}-R_{r}$-completely reducible by Proposition $\alpha(\mathrm{i})$ (over $H$ ). Since the center of $V^{\prime}$ coincides with the center of $R^{\prime}$ which is a field, $V^{\prime}$ is a simple ring, and $\left[R^{\prime}: H\right]=\left[V: V^{\prime}\right]$ is a direct consequence of Theorem 1 . (ii) If suffices to prove that $R$ is $V_{l}^{\prime}-R_{r}$-completely reducible. Since $R$ is $H_{r} V_{r}$ - $R_{l}$-irreducible, $\left[R^{\prime}: H\right]_{l} \leqq\left[V: V^{\prime}\right]_{r}$ (Lemma 1). Set $R^{\prime \prime}=R^{\prime}\left[\left\{e_{i j}\right\}\right]$ and $V_{R}\left(R^{\prime \prime}\right)$ $=V^{\prime \prime}$. Then, by Theorem 1 , we have $\left[R^{\prime \prime}: H\right]=\left[V: V^{\prime \prime}\right]<\infty$. We set here $V^{*}=\sum V d_{h k} \quad$ (simple), where $H=\sum K d_{h k}$. Then, $\left[R^{\prime \prime}: K\right]=\left[V^{*}: V^{\prime \prime}\right]$, $V_{R}\left(V^{*}\right)=K$ and $V_{R}\left(V^{\prime \prime}\right)=R^{\prime \prime}$. Since $R$ is $H_{l} V_{l}-R_{r}$-irreducible, $R$ is also $K_{l} V_{l}^{*}-R_{r}$-irreducible. Noting here $V_{R}\left(V^{*}\right)=K$ (division ring), $R$ is $V_{l}^{*}$ $R_{r}$-irreducible (Lemma 1). Hence, by Proposition $\gamma(\mathrm{ii}), \operatorname{Hom}_{V_{l}}\left(V^{*}, R\right)$ $=\left(\left\langle R^{\prime \prime}\right\rangle \mid V^{*}\right) R_{r}=\sum_{i}\left(\sigma_{i} \mid V^{*}\right) R_{r}$ (direct sum) with some $\sigma_{i}^{\prime}$ s $\in\left\langle R^{\prime \prime}\right\rangle$, where one may remark that each $\left(\sigma_{i} \mid V^{*}\right) R_{r}$ is (left) $V_{r}^{*}$-(right) $R_{r}$-irreducible, 
and is (module) isomorphic to $R$ under the mapping $x \leftrightarrow\left(\sigma_{i} \mid V^{*}\right) x_{r}$. Accordingly, the (left) $V_{r}^{*}$-(right) $R_{r}$-submodule $\operatorname{Hom}_{V_{l}^{\prime}}\left(V^{*}, R\right)$ of $\operatorname{Hom}_{V_{l}^{\prime \prime}}\left(V^{*}, R\right)$ is completely reducible: $\operatorname{Hom}_{V_{l}^{\prime}}\left(V^{*}, R\right)=\sum_{1}^{s} \mathfrak{M i}_{j}$ (direct sum), and each $\because l_{j}$ is (left) $V_{r-}^{*}$ (right) $R_{r}$-isomorphic to $\left(\sigma_{j}^{\prime} \mid V^{*}\right) R_{r}$ for some $\sigma_{j}^{\prime} \in\left\{\sigma_{i}\right\}$. If $\boxplus I_{j} \ni \epsilon_{j} \leftrightarrow \sigma_{j}^{\prime} \mid V^{*} \in\left(\sigma_{j}^{\prime} \mid V^{*}\right) R_{r}$ under the above isomorphism, then, $x_{r}^{*} \epsilon_{j}$ $\leftrightarrow x_{r}^{*}\left(\sigma_{j}^{\prime} \mid V^{*}\right)=\left(\sigma_{j}^{\prime} \mid V^{*}\right)\left(x^{*} \sigma_{j}^{\prime}\right)_{r} \leftrightarrow \epsilon_{j}\left(x^{*} \sigma_{j}^{\prime}\right)_{r}$ for each $x^{*} \in V^{*}$. We have, then, $x_{r}^{*} \epsilon_{j}=\epsilon_{j}\left(x^{*} \sigma_{j}^{\prime}\right)_{r}$, whence, it follows $x^{*} \epsilon_{j}=\left(1 \epsilon_{j}\right)\left(x^{*} \sigma_{j}^{\prime}\right)$. Hence, $\left.\mathfrak{M N}\right\}_{j}$ $=\epsilon_{j} R_{r}=\left(\sigma_{j}^{\prime} u_{j l} \mid V^{*}\right) R_{r}$ with $u_{j}=1 \epsilon_{j}$. Further, for any $x^{*} \in V^{\prime}$, we have $x^{*} \epsilon_{j}=x^{*}\left(1 \epsilon_{j}\right)=x^{*} u_{j}$ and $x^{*} \epsilon_{j}=u_{j}\left(x^{*} \sigma_{j}^{\prime}\right)$, and so, $x^{*} u_{j}=u_{j}\left(x^{*} \sigma_{j}^{\prime}\right)$.

Now, let $V^{\prime} a R\left(a \in V^{*}\right)$ be a $V_{l}^{\prime}-R_{r}$-submodule of $R$ such that the composition series of $V^{\prime} a R$ as $R$-right module is of the shortest length among the $V_{l}^{\prime}-R_{r}$-submodules $\left\{V^{\prime} a R ; a \in V^{*}\right\}$. Then, $V^{\prime} a R$ is a $K_{l} V_{l}^{\prime}-$ $R_{r}$-module. If $M^{\prime}$ is an arbitrary minimal $K_{l} V_{l}^{\prime}-R_{r}$-submodule of $V^{\prime} a R$, then $M^{\prime}=e^{\prime} R\left(e^{\prime} \in V^{*}\right)$ as a submodule of a $K_{l}-R_{r}$-completely reducible module $R$ (note that if $R=M^{\prime} \oplus M^{\prime \prime}$ for some $K_{l}-R_{r}$-module $M^{\prime \prime}$, then $1=e^{\prime}+e^{\prime \prime}$ and $\left.e^{\prime}, e^{\prime \prime} \in V_{R}(K)=V^{*}\right)$, and so, $V^{\prime} a R \supset M^{\prime}=V^{\prime} e R$. We have, therefore, $V^{\prime} a R=V^{\prime} e R=M^{\prime}$. This implies that $V^{\prime} a R$ is a irreducible $K_{l} V_{l}^{\prime}-R_{r}$-submodule of $R$. For an irreducible $V_{l}^{\prime}-R_{r}$-submodule $V^{\prime} x R$ of $V^{\prime} a R$, we have $V^{\prime} a R=K V^{\prime} x R=\sum_{y \in K} V^{\prime} y x R$. It follows that $V^{\prime} a R$ $=\sum_{1}^{n} V^{\prime} x_{i} R$ (direct sum), where $V^{\prime} x_{i} R$ is $V_{l}^{\prime}-R_{r}$-irreducible.

Since $V^{\prime} a \subset V^{*}$, we have

$$
\begin{aligned}
\left(V^{\prime} a\right) \mathfrak{M}_{j} & =\left(V^{\prime} a\right) \sigma_{j}^{\prime} u_{j l} R_{r}=u_{j}\left(\left(V^{\prime} a\right) \sigma_{j}^{\prime}\right) R \\
& \left.=u_{j}\left(\left(V^{\prime} a\left(R \sigma_{j}^{\prime}\right)^{-1}\right)\right) \sigma_{j}^{\prime}\right)=u_{j}\left(\left(V^{\prime} a R\right) \sigma_{j}^{\prime}\right) .
\end{aligned}
$$

Noting that $R$ is $V_{l}^{\prime}-\operatorname{Hom}_{V_{l}}(R, R)$-irreducible, there holds that

$$
\begin{aligned}
R & =\left(V^{\prime} a\right) \operatorname{Hom}_{V_{1}}(R, R)=\left(V^{\prime} a\right)\left(\operatorname{Hom}_{V_{l}}(R, R) \mid V^{*}\right) \\
& =\left(V^{\prime} a\right) \operatorname{Hom}_{V_{l}}\left(V^{*}, R\right)=\left(V^{\prime} a\right) \sum_{1}^{s} \mathfrak{M}_{j}=\sum_{1}^{s}\left(V^{\prime} a\right) \mathfrak{M}_{j} \\
& =\sum_{1}^{s} u_{j}\left(\left(V^{\prime} a R\right) \sigma_{j}^{\prime}\right)=\sum_{1}^{s} u_{j}\left(\left(\sum_{1}^{n} V^{\prime} x_{i} R\right) \sigma_{j}^{\prime}\right) \\
& =\sum_{j=1}^{s} \sum_{i=1}^{n} u_{j}\left(\left(V^{\prime} x_{i} R\right) \sigma_{j}^{\prime}\right) .
\end{aligned}
$$

Finally, we shall show that $u_{j}\left(\left(V^{\prime} x_{i} R\right) \sigma_{j}^{\prime}\right)$ is 0 or a $V_{l}^{\prime}-R_{r}$-irreducible module for every $i, j$. Since $V^{\prime} x_{i} R$ is $V_{l}^{\prime}-R_{r}$-irreducible, $\left(V^{\prime} x_{i} R\right) \sigma_{j}^{\prime}$ is $\left(V^{\prime} \sigma_{j}^{\prime}\right)_{l^{-}}$ $R_{r}$-irreducible. Noting here $u_{j}\left(x^{*} \sigma_{j}^{\prime}\right)=x^{*} u_{j}$ for any $x^{*} \in V^{\prime}$, the mapping $\left(V^{\prime} x_{i} R\right) \sigma_{j}^{\prime}=V^{\prime} \sigma_{j}^{\prime}\left(x_{i} \sigma_{j}^{\prime}\right) R \rightarrow u_{j}\left(V^{\prime} \sigma_{j}^{\prime}\right)\left(x_{i} \sigma_{j}^{\prime}\right) R=V^{\prime} u_{j}\left(x_{i} \sigma_{j}^{\prime}\right) R$ is a right $R$-linear and left $V^{\prime} \sigma_{j}^{\prime}$-semilinear homomorphism. Hence $u_{j}\left(\left(V^{\prime} x_{i} R\right) \sigma_{j}^{\prime}\right)$ is 0 or a $V_{l}^{\prime}$ - $R_{r}$-irreducible module.

REMARK. Let $R$ satisfy $\left(\mathrm{A}_{l}\right)$ over $S$. If (S)' is a $\left(*_{f}\right)$-regular subgroup of $G$, 
then $J\left(\Theta^{\prime}, R\right) \subset J\left(\left\langle V\left(\mathbb{S}^{\prime}\right)\right\rangle, R\right)=V_{R}\left(V\left(\mathbb{S}^{\prime}\right)\right)$ and so

$$
V\left(\aleph^{\prime}\right)=V_{R}\left(J\left(\uplus^{\prime}, R\right)\right) \supset V_{R}^{2}\left(V\left(\uplus^{\prime}\right)\right) \supset V\left(\aleph^{\prime}\right) \text {. }
$$

This implies that $V_{R}^{2}\left(V\left(\mathscr{S}^{\prime}\right)\right)=V\left(\mathscr{S}^{\prime}\right)$. Hence, by Lemma 3 , we have $\left[V: V\left(\Theta^{\prime}\right)\right]_{r}=\left[V: V\left(\bigotimes^{\prime}\right)\right]_{l}$. In particular, if (\$) is $f$-regular, then $\left[V: V\left(\$^{\prime}\right)\right]_{r}=\left[V: V\left(\Theta^{\prime}\right)\right]_{l}$. On the other hand, if $S^{\prime}$ is an $f$-regular subring of $R$, then $V_{R}^{2}\left(V_{R}\left(S^{\prime}\right)\right)=V_{R}\left(S^{\prime}\right)$. Hence, by Lemma 3 , we have $\left[V: V_{R}\left(S^{\prime}\right)\right]_{r}=\left[V: V_{R}\left(S^{\prime}\right)\right]_{l}$.

LEMma 4. Let $R$ satisfy $\left(\mathrm{A}_{l}\right)$ over $S$. If $(S)$ is a subgroup of $B$ such that $\left[J\left(\mathbb{S}^{\prime} \cap \mathbb{S}^{\prime}(H, R), R\right): H\right]_{l}<\infty$, then $\left[J\left(\mathcal{S}^{\prime}, R\right): J\left(\mathcal{S}^{\prime}, R\right) \cap H\right]_{l}<\infty$.

Proof. Set $\mathcal{S}^{\prime \prime}=\left\{\sigma \in \mathbb{S}^{\prime} ; d_{h k} \sigma=d_{h k}\right.$ for each $\left.d_{h k} \in\left\{d_{h k}\right\}\right\}$. Then (ङ)': (S)") $\leqq \prod_{d h k} \#\left\{d_{h k} \sigma ; \sigma \in\left(S^{\prime}\right\}<\infty\right.$. Hence, by Proposition $\beta$,

$$
\left[J\left(\mathcal{S}^{\prime \prime}, R\right) \cap H: J\left(\mathcal{S}^{\prime}, R\right) \cap H\right] \leqq\left(\mathcal{S}^{\prime}: \mathbb{S}^{\prime \prime}\right)<\infty .
$$

Since $J(S), R) \supset\left\{d_{h k}\right\}$, it follows (Lemma 2) that

$$
\begin{aligned}
& \left.\left[J\left(\Theta^{\prime \prime}, R\right): J\left(\Theta^{\prime \prime}, R\right) \cap H\right]_{l}=[H J(\$), R): H\right]_{l} \\
& \leqq\left[J\left(\Theta^{\prime \prime} \cap \Theta(H, R), R\right): H\right]_{l}=\left[J\left(\Theta^{\prime} \cap \circlearrowleft(H, R), R\right): H\right]_{l}<\infty
\end{aligned}
$$

(note that $\mathcal{S}^{\prime \prime} \cap \mathcal{S}(H, R)=\mathcal{S}^{\prime} \cap \mathcal{B}(H, R)$ ). Hence,

$$
\begin{aligned}
& {\left[J\left(\oiint^{\prime}, R\right): J\left(\oiint^{\prime}, R\right) \cap H\right]_{l} \leqq\left[J\left(\mathbb{S}^{\prime \prime}, R\right): J\left(\mathbb{S}^{\prime}, R\right) \cap H\right]_{l}} \\
& =\left[J\left(\$^{\prime \prime}, R\right): J\left(\oiint^{\prime \prime}, R\right) \cap H\right]_{l}\left[J\left(\oiint^{\prime \prime}, R\right) \cap H: J\left(\oiint^{\prime}, R\right) \cap H\right]_{l}<\infty .
\end{aligned}
$$

This completes the proof.

Remark. Let $R$ satisfy $\left(\mathrm{A}_{l}\right)$ over $S$. If $(S)^{\prime}$ is a subgroup of $(S)$, then $J\left(\mathcal{S}^{\prime}, R\right) \cap H$ is $f$-regular. By Corollary $4, R$ is left locally finite over $J\left(\mathcal{S}^{\prime}, R\right) \cap H$. Since $R$ is $S_{l} V_{l^{-}} R_{r^{-}}$-irreducible, $R$ is $\left(J\left(\mathcal{S}^{\prime}, R\right) \cap H\right)_{l} V_{l^{-}} R_{r^{-}}$ irreducible. Hence $R$ satisfies $\left(\mathrm{B}_{l}\right)$ (and so $\left(\mathrm{A}_{l}\right)$ ) over $J\left(\Theta^{\prime}, R\right) \cap H$. If, more-

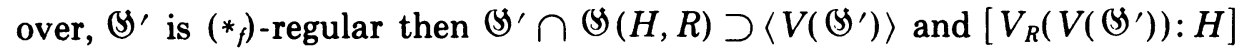
$<\infty$ (Lemma 3). Hence, by Lemma 4, $\left[J\left(\Theta^{\prime}, R\right): J\left(\Theta^{\prime}, R\right) \cap H\right]_{l}<\infty$. As in $[4$, p. 84], we may place the finite topology on the group $(\$)$. Here a basis for the neighborhoods of $\sigma \in \Theta$ consists of the sets $U(\sigma, F)$ $=\{\tau \in G ; \tau|F=\sigma| F\}$, where $F$ runs over all the (nonempty) finite subsets of $R$ (or subrings of $R$ finitely generated over $S$ ). Then (S) is a topological group which is totally disconnected, and $U(\sigma, F)$ is not only open but also closed. If $\mathbb{S}^{\prime}$ ' is an $f$-regular subgroup of $(S)$, then $J\left(\mathbb{S}^{\prime}, R\right)$ is an $f$-regular subring with $\left[J\left(\mathcal{S}^{\prime}, R\right): J\left(\mathcal{S}^{\prime}, R\right) \cap H\right]_{l}<\infty$. Hence, by Theorem $1, R$ satisfies $\left(\mathrm{A}_{l}\right)$ over $J\left(\Theta^{\prime}, R\right)$. Moreover, by Remark 1 of Theorem $1, \mathcal{S}^{\prime}\left|F=\mathcal{S}^{\prime}\left(J\left(\mathcal{S}^{\prime}, R\right), R\right)\right| F$ for any finite subset $F$ of $R$. Since $(S)\left(S\left(S^{\prime}, R\right), R\right)=\bigcap \circlearrowleft(S[F], R)$ ( $F$ runs over all the (nonempty) finite subsets of $\left.J\left(\xi^{\prime}, R\right)\right),()^{\prime}\left(J\left(\xi^{\prime}, R\right), R\right)$ is a closed subgroup of $G$. Hence $\Theta^{\prime}$ is dense in the closed $f$-regular subgroup $(S)\left(J\left(\mathscr{S}^{\prime}, R\right), R\right)$. In particular, if $\mathcal{S}^{\prime}$ is a closed $f$-regular subgroup of $(B)$, then $(S)\left(J\left(S^{\prime}, R\right), R\right)=\left(\mathcal{S}^{\prime}\right.$. 
Lemma 5. Let $R$ satisfy $\left(\mathrm{A}_{l}\right)$ over $S$. If $\left(S^{\prime}\right.$ is a closed f-regular subgroup of $(S)$ then $\mathbb{S}^{\prime}\langle V\rangle$ is a closed f-regular subgroup of $(S)$ and so

$$
\text { (S)' }\langle V\rangle=\text { (S) }\left(H \cap J\left(S^{\prime}, R\right), R\right) \text {. }
$$

Proof. Set $S^{\prime}=J\left(S^{\prime}, R\right)$ and $H^{\prime}=H \cap S^{\prime}$. Then, by the remark of Lemma $4, R$ satisfies $\left(\mathrm{A}_{l}\right)$ over $H^{\prime}$ and $\left[S^{\prime}: H^{\prime}\right]<\infty$. Set $T=H^{\prime}\left[S^{\prime},\left\{e_{i j}\right\}\right]$. Then $T$ is a regular subring of $R$ with $\left[T: H^{\prime}\right]<\infty$. Hence, $[T: H \cap T]$ $=\left[V: V_{R}(T)\right]$, and $R$ satisfies $\left(\mathrm{A}_{l}\right)$ over $H \cap T$ (Theorem 1). Noting here $T \supset\left\{e_{i j}\right\}, R$ is $T_{l}-R_{r}$-irreducible. Hence, by Proposition $\gamma(\mathrm{ii})$, we have (S) $(H \cap T, R)|T=\langle V\rangle| T$. This implies that $\$(H \cap T, R)=\mathbb{S}(T, R)\langle V\rangle$. Since $J\left(\mathcal{S}^{\prime} \mid H, H\right)=H^{\prime}$ and $\left[H \cap T: H^{\prime}\right]<\infty$, (S) $\left(H^{\prime}, R\right)\left|H \cap T=\mathcal{S}^{\prime}\right| H$ $\cap T$ (Proposition $\beta$ and its remark). Hence, for $\mathcal{S}^{\prime} \mid H \cap T=\left\{\sigma_{i} \mid H \cap T\right.$; $\left.i=1, \cdots, n=\left[H \cap T: H^{\prime}\right]\right\}\left(\sigma_{i} \in\left(^{\prime}\right)\right.$, we have

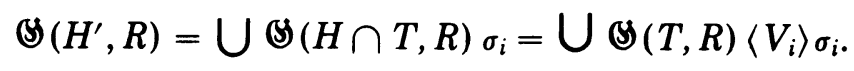

Since $S^{\prime}$ is closed and $f$-regular, $S^{\prime}=\mathbb{S}\left(J\left(\mathcal{S}^{\prime}, R\right), R\right)=\Theta\left(S^{\prime}, R\right)$ by the remark of Lemma 4 , and from this it follows that $(S) R) \subset \mathbb{B}(T$. This implies that $\mathbb{S}\left(H^{\prime}, R\right)=\bigcup \mathbb{S}(T, R)\langle V\rangle \sigma_{i} \subset \mathbb{S}^{\prime}\langle V\rangle$. On the other hand, (S) $\left(H^{\prime}, R\right) \supset\left(S^{\prime}\langle V\rangle\right.$. Hence, $\&\left(H^{\prime}, R\right)=\left(S^{\prime}\langle V\rangle\right.$, which is a closed $f$-regular subgroup of (S).

Lemma 6. Let $R$ satisfy $\left(\mathrm{A}_{l}\right)$ over $S$. If an $N$-regular subgroup (S) of (S) contains a closed $f$-regular subgroup $\left(S^{*}\right.$ such that $I\left(\mathbb{S}^{\prime}\right)=I\left(\mathbb{S}^{*}\right)\left(=V\left(\mathbb{S}^{*}\right)\right)$ and $\left(\mathbb{S}^{\prime}: \mathbb{S}^{*}\right)<\infty$, then $\mathfrak{S}^{\prime}$ is closed and f-regular.

Proof. Set $S^{\prime}=J\left(\mathcal{S}^{\prime}, R\right)$ and $S^{*}=J\left(\xi^{*}, R\right)$. Then, by Lemma 5 , $(\xi)\left(H \cap S^{*}, R\right) \cap S^{\prime}=\left(S^{*}\langle V\rangle \cap S^{\prime}\right.$. For any $\sigma$ in this intersection, we may write $\sigma=\tau\langle a\rangle, \quad \tau \in\left(\mathbb{S}^{*}, \quad\langle a\rangle \in\langle V\rangle\right.$. Then $\langle a\rangle=\tau^{-1} \sigma \in\left\langle I\left(\mathbb{S}^{\prime}\right)\right\rangle$ $=\left\langle I\left(\mathbb{S}^{*}\right)\right\rangle \subset \mathbb{S}^{*}$ and so $\sigma=\tau\langle a\rangle \in \mathbb{S}^{*}$. This implies that $\mathbb{S}^{*}\left(H \cap S^{*}, R\right)$ $\cap \mathcal{S}^{\prime}=\mathcal{S}^{*}$. If, for $\sigma$ in $\mathcal{S}^{\prime}, \sigma \mid H \cap S^{*}$ is the identity, then $\sigma$ is in $(H)\left(H \cap S^{*}, R\right)$ $\cap \mathscr{S}^{\prime}=\left(^{*}\right.$, so that $\sigma \mid S^{*}$ is the identity. It follows that for an automorphism $\sigma$ in $\mathcal{H}^{\prime}, \sigma \mid S^{*}$ is the identity if and only if $\sigma \mid H \cap S^{*}$ is the identity, and that

$$
\infty>\left(\mathbb{S}^{\prime}: \mathbb{S}^{*}\right)=\#\left(\mathbb{S}^{\prime} \mid S^{*}\right)=\#\left(\mathbb{S}^{\prime} \mid H \cap S^{*}\right)=\left[H \cap S^{*}: H \cap S^{\prime}\right]
$$

(Proposition $\beta$ ). Now, let $S^{* *}$ be a subring generated by $\bigcup_{\sigma \in()^{\prime}} S^{*} \sigma$. Then, noting that $\left\langle I\left(\left(^{\prime}\right)\right\rangle\right.$ is a normal subgroup of ${\left(S^{\prime}\right.}^{\prime}$ and $S^{*} \subset V_{R}\left(I\left(S^{*}\right)\right)$ $=J\left(\left\langle I\left(\mathbb{S}^{\prime}\right)\right\rangle, R\right), S^{* *}$ is a subring of $V_{R}\left(I\left(B^{*}\right)\right)$ containing $S^{*}$. By the remark of Lemma $4, R$ satisfies $\left(\mathrm{A}_{l}\right)$ over $S^{*}$. Hence, $S^{* *}$ is simple by Proposition $\beta$ and its remark. Moreover, we have \#(B)' $\left.\mid S^{* *}\right) \leqq\left(\mathcal{S}^{\prime}: \mathcal{S}^{*}\right)^{2}$ $<\infty$. If, for an automorphism $\sigma$ in $\left(S^{\prime}, \sigma \mid S^{* *}\right.$ is an inner automorphism $\langle a\rangle$ of $S^{* *}$, then, $\sigma\left|S^{*}=\langle a\rangle\right| S^{*}$ and $\langle a\rangle \in\langle V\rangle$, so that $\sigma \mid H \cap S^{*}$ is the identity. Hence, $\sigma \mid S^{*}$ is the identity an so $a$ is in $V_{R}\left(S^{*}\right)=I\left(S^{*}\right)$. Noting here $S^{* *} \subset V_{R}\left(I\left(S^{*}\right)\right),\langle a\rangle \mid S^{* *}$ is the identity. Thus $\left(^{\prime}\right) \mid S^{* *}$ is a 
finite group of outer automorphisms of $S^{* *}$, and, hence, $S^{* *}$ is finite outer

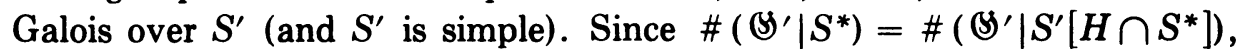
we have $S^{*}=S^{\prime}\left[H \cap S^{*}\right]$ (Proposition $\beta$ ). This implies that $V_{R}\left(S^{\prime}\right)$ $=V_{R}\left(S^{*}\right)=I\left(S^{*}\right)=I\left(S^{\prime}\right)$, so that $S^{\prime}$ is $f$-regular. Further, noting that ((S): (S)*) $<\infty, \mathbb{S}^{\prime}$ is closed.

Theorem 2. Let $R$ satisfy $\left(\mathrm{A}_{l}\right)$ over $S$. Let $\left(\mathbb{S}^{\prime}\right.$ be a closed $N_{f}$-regular subgroup of $\left(\mathcal{S}^{\prime}\right.$ such that $V_{R}^{2}\left(I\left(\mathcal{S}^{\prime}\right)\right)=I\left(\mathcal{S}^{\prime}\right)$. Then $\mathbb{B}^{\prime}$ is f-regular if and only if $\left(\mathbb{S}^{\prime}:\left(\mathbb{S}_{1} \cap \mathbb{S}^{\prime}\right)\left\langle I\left(\mathbb{S}^{\prime}\right)\right\rangle\right)<\infty$ for every open subgroup $\mathbb{S}_{i}$ of $\mathbb{S}^{\prime}$.

Proof. Suppose that $\mathcal{S}^{\prime}$ is $f$-regular. Let $\mathcal{H}^{\prime}$, be an open subgroup of $(S)$. Then there exists a finite subset $F$ such that $(S)(F], R) \subset(B)$ (note that, for $\sigma$ in $(S)$ the sets $U(\sigma, F)=\{\tau \in(S),|F=\sigma| F\}$ ( $F$ runs over all the (nonempty) finite subsets of $R$ ) is a basis for the neighborhoods of $\sigma$ ). Set $S^{\prime}=J\left(S^{\prime}, R\right)$. Then, by the remark of Lemma $4, R$ satisfies $\left(\mathrm{A}_{l}\right)$ over $S^{\prime}$. Set $T=S^{\prime}\left[F,\left\{e_{i j}\right\}\right]$. Then $\left[T: S^{\prime}\right]<\infty$, and $(S(T, R)$ is a closed $f$ regular subgroup of $\left(S\left(S^{\prime}, R\right)\right.$ such that $J(S(T, R), R)=T$. Hence, by Lemma 5 (for $S=S^{\prime}$ and $\left.H=V_{R}^{2}\left(S^{\prime}\right)\right)$, $\left(V_{R}^{2}\left(S^{\prime}\right) \cap T, R\right)=\$(T, R)$ $\left\langle V_{R}\left(S^{\prime}\right)\right\rangle$. Further, we have

$$
\begin{aligned}
& \text { (\$) }\left(S^{\prime}, R\right): \text { S }\left(V_{R}^{2}\left(S^{\prime}\right) \cap T, R\right)=\#\left(\$\left(S^{\prime}, R\right) \mid V_{R}^{2}\left(S^{\prime}\right) \cap T\right) \\
& =\left[V_{R}^{2}\left(S^{\prime}\right) \cap T: S^{\prime}\right]<\infty
\end{aligned}
$$

(Proposition $\beta$ and its remark). We have, therefore,

$$
\text { (\$) } \left.\left(S^{\prime}, R\right): \text { (S }(T, R)\left\langle V_{R}\left(S^{\prime}\right)\right\rangle\right)<\infty \text {. }
$$

Then, noting that $(\mathcal{S})(T, R) \subset\left({ }^{\prime}\right)\left(S^{\prime}, R\right)=\left(\xi^{\prime}\right.$ and $V_{R}\left(S^{\prime}\right)=I\left(\mathcal{S}^{\prime}\right)$, and that (S) $(T, R) \subset(S)(S[F], R) \subset\left(^{(S)}\right.$, it follows that $\left(\left(^{\prime}\right):\left(\mathcal{S}_{l} \cap\left(S^{\prime}\right)\left\langle I\left(\mathcal{S}^{\prime}\right)\right\rangle\right)<\infty\right.$.

Suppose that $\left.\left(\mathbb{S}^{\prime}: \mathbb{S}_{1} \cap \mathbb{S}^{\prime}\right)\left\langle I\left(\mathbb{S}^{\prime}\right)\right\rangle\right)<\infty$ for every open subgroup (S), of $\mathbb{S}^{(S) \text { Since }} V_{R}^{2}\left(I\left(\mathbb{S}^{\prime}\right)\right)=I\left(\mathbb{S}^{\prime}\right)$ and $\left[V: I\left(\mathbb{S}^{\prime}\right)\right]_{\gamma}<\infty$, there exists a finite subset $F$ of $V_{R}\left(I\left(\mathbb{S}^{\prime}\right)\right)$ such that $V_{R}(S[F])=I\left(\mathbb{S}^{\prime}\right)$. Further, by Lemma 3, $V_{R}\left(I\left(\mathbb{S}^{\prime}\right)\right)$ is simple. Let $\left\{d_{h k}^{\prime}\right\}$ be matrix units of $V_{R}\left(I\left(\mathbb{(}^{\prime}\right)\right)$ such that the centralizer of $\left\{d_{h k}^{\prime}\right\}$ in $V_{R}\left(I\left(S^{\prime}\right)\right)$ is a division ring. Set here $S^{*}=\mathbb{S}\left(S\left[F,\left\{d_{h k}^{\prime}\right\}\right], R\right)$. Then $\mathbb{S}^{*}$ is closed, and so $\mathscr{S}^{*} \cap \mathbb{S}^{\prime}$ is a closed $f$-regular subgroup of $(S)$ such that $I\left(S^{*} \cap\left(S^{\prime}\right)=I\left(\mathcal{S}^{\prime}\right)\right.$. On the other hand, $\mathbb{S}^{*}$ is open. Then, noting here $\mathbb{S}^{*} \cap \mathbb{S}^{\prime} \supset\left\langle I\left(\mathbb{S}^{\prime}\right)\right\rangle$, we have $\left(\mathbb{S}^{\prime}:\left(\mathbb{S}^{*} \cap \mathcal{S}^{\prime}\right)\right)<\infty$. Hence, by Lemma 6, $\mathfrak{S}^{\prime}$ is $f$-regular.

Corollary 5. Let $R$ satisfy $y^{3}\left(A_{l}\right)$ over $S$. Let $V$ be finite over the center $C$ of $R$, and set $S^{*}=\mathbb{S}\left(S\left[\left\{e_{i j}\right\},\left\{r_{v}\right\}\right], R\right)$, where $\left\{r_{v}\right\}$ is a linearly independent left basis of $R$ over $H$. Let (\$)' be a closed $N$-regular subgroup of (\$). Then, the following conditions are all equivalent.

(i) ()$^{\prime}$ is regular.

(ii) $\left(\mathbb{S}^{\prime}:\left(\mathbb{S}^{*} \cap \mathbb{S}^{\prime}\right)\left\langle I\left(\mathbb{S}^{\prime}\right)\right\rangle\right)<\infty$.

(iii) $\left(\mathbb{S}^{\prime}:\left(\mathbb{S}_{1} \cap \mathbb{S}^{\prime}\right)\left\langle I\left(\mathbb{S}^{\prime}\right)\right\rangle\right)<\infty$ for every open subgroup $\mathbb{S}_{\text {, of }}\left(\mathbb{S}^{\prime}\right.$. 
Proof. Since [ $V: C]<\infty$, (S)' is an $N_{f}$-regular subgroup of (S) such that $V_{R}^{2}\left(I\left(\mathbb{S}^{\prime}\right)\right)=I\left(\mathbb{S}^{\prime}\right)$ (the fundamental theorem of simple rings). Further (\$) ${ }^{*}$ is an open (closed) subgroup of (S). Hence, by Theorem 2 , it suffices to prove that (ii) $\Longrightarrow$ (iii). Let $(S)$ be an open subgroup of $(S)$. Then there exists a finite subset $F$ of $R$ such that $(S)(F], R) \subset(S)$. Since $\mathbb{S}^{*}$ is closed, (S) $\cap \xi^{\prime}$ is closed and outer (and regular). Set $T=J\left(\xi^{*} \cap \mathbb{S}^{\prime}, R\right.$ ) (regular). Then $\mathscr{S}^{*} \cap \mathbb{S}^{\prime}=\mathscr{S}(T, R)$ (the remark of Lemma 4). Noting here $[T[F]: T]<\infty$, we have $\left(\mathbb{S}^{*} \cap \mathbb{S}^{\prime}: \mathbb{S}(T[F], R)\right)=\left(\left(\mathbb{S}^{*} \cap \mathbb{S}^{\prime}\right) \mid T[F]\right)$ $=[T[F]: T]<\infty \quad($ Proposition $\beta)$. Hence

$$
\left(\left(\mathbb{S}^{*} \cap \mathbb{S}^{\prime}\right)\left\langle I\left(\mathbb{S}^{\prime}\right)\right\rangle: \mathbb{S}(T[F], R)\left\langle I\left(\mathbb{S}^{\prime}\right)\right\rangle\right)<\infty \text {, }
$$

and so

$$
\begin{aligned}
\left(\mathbb{S}^{\prime}:\right. & \left.\mathbb{S}(T[F], R)\left\langle I\left(\mathbb{S}^{\prime}\right)\right\rangle\right) \\
\quad= & \left(\mathbb{S}^{\prime}:\left(\mathbb{S}^{*} \cap \mathbb{S}^{\prime}\right)\left\langle I\left(\mathbb{S}^{\prime}\right)\right\rangle\right)\left(\left(\mathbb{S}^{*} \cap \mathbb{S}^{\prime}\right)\left\langle I\left(\mathbb{S}^{\prime}\right)\right\rangle: \mathbb{S}(T[F], R)\left\langle I\left(\mathbb{S}^{\prime}\right)\right\rangle\right) \\
< & \infty .
\end{aligned}
$$

Since (") $(T[F], R) \subset($ () $(S[F], R) \subset$ ("), and (") $(T[F], R) \subset$ (") $(T, R)=($ () * $\cap()^{\prime} \subset()^{\prime}$, it follows that

$$
\left(()^{\prime}:\left(()_{1} \cap\left(^{\prime}\right)\left\langle I\left(\left(^{\prime}\right)\right\rangle\right)<\infty\right. \text {. }\right.
$$

Remark. The equivalence (i) $\Longleftrightarrow$ (ii) is contained in [4]. Further, Corollary 5 is still true for replacing the condition that $V$ is finite over the center of $R$ by the condition that $(y)$ is locally compact, and $(H *$ by a compact closed-open subgroup of $(H)$ respectively. The proof may be left to the reader. In case that $V$ is finite over the center of $R$, there exists a counter example of Galois extension satisfying $\left(\mathrm{A}_{l}\right)$ over $S$ such that $(S)$ contains a closed $N$-regular subgroup of (S) which is not regular [4, Introduction].

TheOREm 3. Let $R$ satisfy $\left(\mathrm{A}_{l}\right)$ over $S$. Then, every $\left(*_{f}\right)$-regular subgroup (5)' of (s) is f-regular.

Proof. Set $S^{\prime}=J\left(\mathbb{S}^{\prime}, R\right)$. Then $V_{R}\left(S^{\prime}\right)=V\left(\xi^{\prime}\right)=I\left(\mathbb{S}^{\prime}\right)=V_{R}^{2}\left(I\left(\mathbb{S}^{\prime}\right)\right)$ (Definition). Hence, it suffices to prove that $\mathcal{S}^{\prime}\left(S^{\prime}, R\right)$ is $f$-regular. By the remark of Lemma $4, R$ satisfies $\left(\mathrm{A}_{l}\right)$ over $H \cap S^{\prime}$, and $\left[S^{\prime}: H \cap S^{\prime}\right]_{l}<\infty$; i.e., $S^{\prime}=\left(H \cap S^{\prime}\right)[F]$ for some finite subset $F$ of $S^{\prime}$. Let $\left(S_{\text {, be an open }}\right.$ subgroup of (s). Then (S) $(S[F], R) \cap$ (l), is also an arbitrary open subgroup of ( $($ ). Hence, by Theorem 2, we have

$$
\text { (\$) } \left.\left(H \cap S^{\prime}, R\right):\left(\mathbb{S}\left(H \cap S^{\prime}, R\right) \cap(S)(S[F], R) \cap \mathbb{S}_{\triangleleft}\right)\langle V\rangle\right)<\infty \text {. }
$$

Further, we have

$$
\text { (け) }\left(H \cap S^{\prime}, R\right) \supset\left(S^{\prime}, R\right) \text {, }
$$

and 


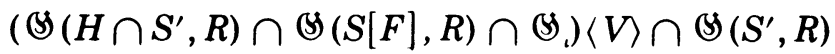

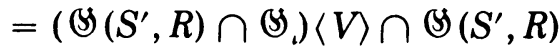

$$
\begin{aligned}
& =\left(\$\left(S^{\prime}, R\right) \cap\left(S_{)}\right)\left\langle I\left(S^{\prime}\left(S^{\prime}, R\right)\right)\right\rangle\right. \text {. }
\end{aligned}
$$

Hence, it follows from (1) and (2) that

$$
\text { (ङ) }\left(S^{\prime}, R\right):\left(\$\left(S^{\prime}, R\right) \cap \mathcal{S}_{1}\right)\left\langle I\left(\left(\text { () }\left(S^{\prime}, R\right)\right)\right\rangle\right)<\infty \text {. }
$$

Therefore by Theorem $2,(S)\left(S^{\prime}, R\right)$ is $f$-regular.

Corollary 6. Let $R$ satisfy $\left(\mathrm{A}_{l}\right)$ over $S$, and let $T$ be a subring of $R$ containing $S$ with $[T: S]_{l}<\infty$. Then, $R$ is $T_{l}$ - $R_{r}$-irreducible if and only if $T$ is simple and $V_{R}(T)$ is a division ring (i.e., $R$ is a regular subring such that $V_{R}(T)$ is a division ring).

Proof. "If" part is a direct consequence of Proposition $\alpha(\mathrm{i})$. For "only if" part, let $R$ be $T_{l}-R_{r}$-irreducible. Then $V_{R}(T)$ is a division ring, and $\left[V: V_{R}(T)\right]_{r}<\infty$ by Corollary 1 . Hence $(S)(T, R)$ is $\left(*_{f}\right)$-regular and so $f$-regular (Theorem 3). By Proposition $\alpha$ (ii), we have $J(\$(T, R), R)=T$. Thus $T$ is simple.

THEOREM 4. Let $R$ satisfy $\left(\mathrm{A}_{l}\right)$ over $S$. Then, the following conditions are equivalent to each other:

(i) Every subring of $R$ containing $S$ is simple.

(ii) $V$ is a division ring.

Proof. (i) $\Longrightarrow$ (ii): For any nonzero element $a$ of $V, S[a]$ is simple, and so, the center of $S[a]$ is a field. Since $a$ is contained in the center of $S[a]$, $a$ is regular (in $R$ ). Hence $V$ is a division ring. (ii) $\Longrightarrow(\mathrm{i})$ : By Corollary $6, R$ is $S_{l}-R_{r}$-irreducible. Hence, for any subring $S^{\prime}$ of $R$ containing $S$ with $\left[S^{\prime}: S\right]_{l}<\infty, R$ is $S_{l}^{\prime}-R_{r}$-irreducible, and so $S^{\prime}$ is simple by Corollary 6 . For any subring $R^{\prime}$ of $R$ containing $S$, we have $R^{\prime}=\bigcup S_{\text {، }}$, where $S_{\text {، }}$ runs over all the subrings of $R^{\prime}$ finite over $S$. Then, noting that the uniquely determined number of $S_{\imath}$-irreducible direct summands of $S_{\iota}$ is not greater than that of $R, R^{\prime}$ is simple.

Let $R$ satisfy $\left(\mathrm{A}_{l}\right)$ over $S$. If a subgroup $\left(\xi^{\prime}\right.$ of $(B)$ is closed and $\left(*_{f}\right)$ regular (and so $f$-regular by Theorem 3 ) then $J\left(S^{\prime}, R\right)$ is $f$-regular and (S) $\left(J\left(\xi^{\prime}, R\right), R\right)=\mathcal{S}^{\prime}$ (and $R$ satisfies $\left(\mathrm{A}_{l}\right)$ over $J\left(\xi^{\prime}, R\right)$ ). Conversely, if a subring $S^{\prime}$ of $R$ containing $S$ is $f$-regular then $(S)\left(S^{\prime}, R\right)$ is closed and $\left(*_{f}\right)$-regular (and so $f$-regular). But we cannot see whether $J\left((S)\left(S^{\prime}, R\right), R\right)$ coincides with $S^{\prime}$ or not. However, in case $[R: H]_{l} \leqq \mathcal{\aleph}_{0}$, we have $J\left(\mathcal{S}^{\prime}\left(S^{\prime}, R\right), R\right)$ $=S^{\prime}$ by the fundamental theorem of [4] and Theorem 1 . Hence, the fundamental theorem takes the place of the next

Theorem 5. Let $R$ satisfy $\left(\mathrm{A}_{l}\right)$ over $S$, and $[R: H]_{l} \leqq \boldsymbol{\aleph}_{0}$. Then there exists a 1-1 dual correspondence between closed $\left(*_{f}\right)$-regular subgroups of $(y)$ 
and f-regular subrings of $R$ containing $S$ in the usual sense of Galois theory. In particular, if $V$ is a division ring, there exists a 1-1 dual correspondence between closed subgroups $\left\{\mathbb{S}^{\prime} ;\left\langle V\left(\mathbb{S}^{\prime}\right)\right\rangle \subset \mathbb{B}^{\prime}\right.$ and $\left.\left[V: V\left(\mathbb{S}^{\prime}\right)\right]<\infty\right\}$ of (s) and subrings $\left\{S^{\prime} ; S^{\prime} \supset S\right.$ and $\left.\left[V: V_{R}\left(S^{\prime}\right)\right]<\infty\right\}$ of $R$, in the usual sense of Galois theory.

Finally, we shall remark about finite Galois theory. So far we have not required the Galois theory of simple rings [6], [7] except for the (finite) outer Galois theory and for the fundamental theorem of simple rings (i.e., the inner Galois theory of simple rings). Moreover, the fundamental theorem is required only for the Remarks 3,4 of Theorem 1 and Corollary 5. If $R$ is finite and Galois over $S$, then $[V: C]<\infty$ (Corollary 1) and $R$ satisfies $\left(D_{l}\right)$ over $S$ (the fundamental theorem of simple rings), and so $\Theta(S, R)$ is an $N$-regular group with $(\circlearrowleft(S, R):\langle I(\circlearrowleft)(S, R))\rangle)[I(\Theta(S, R)): C]$ $(=[R: S])<\infty$ (Proposition $\beta$ and its remark), where $C$ is the center of $R$. Conversely, let $(\$)$ be an $N$-regular group of automorphisms of $R$ with (\$): $\langle I(\mathbb{S})\rangle)[I(\mathbb{S}): C]<\infty$. Set $H^{*}=V_{R}(I(\mathbb{S}))$. Then, by the fundamental theorem of simple rings, $R$ satisfies $\left(\mathrm{D}_{l}\right)$ (and so $\left(\mathrm{B}_{l}\right)$ ) over $H^{*}$ and $\left[R: H^{*}\right]<\infty$. Hence $R$ is $\left.H_{l}^{*} I(\circlearrowleft)\right)_{l} R_{r}$-irreducible, that is, $R$ is a completely reducible homogeneous $I(\$) t_{l}-R_{r}$-module. If $M$ is an $I(\mathbb{S})_{l}-R_{r^{-}}$ submodule of $R$, then this is a direct summand of $R ; R=M+M^{\prime}$ (direct sum) for some $I(\$))_{l}-R_{r}$-submodule $M^{\prime}$ of $R$, and, hence, $M=e R$ for some idempotent $e$ in $H^{*}$. Now we set $\left.S^{*}=J(\Theta), R\right)$. Then, noting that $\Theta\left(H^{*}, R\right)=\langle I(\Theta)\rangle,(\Theta) \mid H^{*}$ is a finite group, of order $(\mathbb{S}:\langle I(\mathbb{S})\rangle)$, of outer automorphisms of $H^{*}$ such that $J\left(\Theta \mid H^{*}, H^{*}\right)=S^{*}$. Hence, by outer Galois theory (Proposition $\beta$ ), $S^{*}$ is simple, and $H^{*}$ is finite and outer Galois over $S^{*}$. Moreover, $H^{*}$ satisfies $\left(\mathrm{D}_{l}\right)$ (and so $\left(\mathrm{B}_{l}\right)$ ) over $S^{*}$. Hence, $H^{*}$ is $S_{l}^{*} V_{H^{*}}\left(S^{*}\right)_{l}-H_{r}^{*}$-irreducible. Let $M$ be an $S_{l}^{*} I(\text { (S) })_{l}-R_{r}$-submodule of $R$. Then, trivially, $M$ is an $I(\$))_{l}-R_{r}$-submodule of $R$, so that $M=e R$ for some idempotent $e$ in $H^{*}$. Noting here $V_{H^{*}}\left(S^{*}\right)=V_{H^{*}}\left(H^{*}\right)$ $C I(\mathbb{S})$, we have $M \supset S^{*} V_{H^{*}}\left(S^{*}\right) e H^{*}=H^{*}$. This implies that $M=R$, that is, $R$ is $S_{l}^{*} I(\circlearrowleft)_{l}-R_{r}$-irreducible, and so, $R$ is $S_{l}^{*} V_{R}\left(S^{*}\right)_{l}-R_{r}$-irreducible. Hence, $V_{R}\left(S^{*}\right)$ is simple, and so, $S^{*}$ is regular. Consequently, $R$ is finite and Galois over $S^{*}$, and $\left[V_{R}\left(S^{*}\right): C\right] \leqq\left[R: S^{*}\right]<\infty$ (Theorem 1). Since $(B)$ is an $N$-regular subgroup of $(B)\left(S^{*}, R\right)$ such that $(\mathbb{S}:\langle I(\mathbb{S})\rangle)[I(\mathbb{S}): C]$ $<\infty$, we have $\mathbb{S}=\mathscr{S}\left(S^{*}, R\right)$ by Corollary 5 and the remark of Lemma 4 (note that $\left(\mathcal{S}^{\prime}\left(S^{*}, R\right)\right.$ is discrete). For any $N$-regular subgroup $\mathcal{S}^{\prime}$ of $\mathcal{S}^{\prime}$, $\left(\mathbb{S}^{\prime}:\left\langle I\left(\mathbb{S}^{\prime}\right)\right\rangle\right)=\left(\mathbb{S}^{\prime}\langle I(\mathbb{(})\rangle:\left\langle I\left(\mathbb{S}^{\prime}\right)\right\rangle\right) \leqq\left(\mathbb{S}^{\prime}:\left\langle I\left(()^{\prime}\right)\right\rangle\right)<\infty$. Hence, $J\left(\mathcal{S}^{\prime}, R\right)$ is regular and $\mathcal{S}^{\prime}\left(J\left(\mathcal{S}^{\prime}, R\right), R\right)=\left(\mathcal{S}^{\prime}\right.$. Moreover, for a regular subring $S^{\prime}$ of $R$ containing $S^{*},(S)\left(S^{\prime}, R\right)$ is $N$-regular, and $\left.J(B)\left(S^{\prime}, R\right), R\right)$ $=S^{\prime}$ by $\left(\mathrm{E}_{l}\right)$. This is the Galois correspondence of finite Galois extensions of simple rings. 


\section{REFERENCES}

1. N. Jacobson, Structure of rings, Amer. Math. Soc. Colloq. Publ. Vol. 37, Amer. Math. Soc., Providence, R. I., 1956.

2. T. Nagahara, On algebraic Galois extensions of simple rings, Math. J. Okayama Univ. 11 (1962), 59-65.

3. T. Nagahara and H. Tominaga, On Galois and locally Galois extensions of simple rings, Math. J. Okayama Univ. 10 (1961), 143-166.

4. __ On Galois theory of simple rings, Math. J. Okayama Univ. 11 (1963), 79-117.

5. - Some theorems on Galois theory of simple rings, J. Fac. Sci. Hokkaido Univ. Ser. I 17 (1963), 1-13.

6. T. Nakayama, Galois theory of simple rings, Trans. Amer. Math. Soc. 73 (1962), 276-292.

7. A. Rosenberg and D. Zelinsky, Galois theory of continuous linear transformation rings, Trans. Amer. Math. Soc. 79 (1955), 429-452.

8. H. Tominaga, Galois theory of simple rings, Math. J. Okayama Univ. 6 (1956), 29-48.

9. , Galois theory of simple rings. II, Math. J. Okayama Univ. 6 (1957), 153-170.

10. J. H. Walter, On the Galois theory of division rings, Proc. Amer. Math. Soc. 10 (1957), 868-907.

\section{NORTHWESTERN UNIVERSITY,}

Evanston, ILunois 